\title{
Guidelines for Diagnosis and Treatment of Carcinoma of the Esophagus April 2012 edited by the Japan Esophageal Society
}

\author{
Hiroyuki Kuwano • Yasumasa Nishimura - Tsuneo Oyama • Hiroyuki Kato · Yuko Kitagawa • \\ Motoyasu Kusano $\cdot$ Hideo Shimada $\cdot$ Hiroya Takiuchi $\cdot$ Yasushi Toh $\cdot$ Yuichiro Doki $\cdot$ Yoshio Naomoto \\ Hisahiro Matsubara • Tatsuya Miyazaki $\cdot$ Manabu Muto $\cdot$ Akio Yanagisawa
}

Received: 18 July 2014 / Accepted: 8 October 2014 / Published online: 11 November 2014

(C) The Japan Esophageal Society and Springer Japan 2014

Keywords Clinical guidelines $\cdot$ Esophagus $\cdot$ Neoplasm

\section{Introduction}

Purpose

These guidelines are intended for doctors who are engaged in the diagnosis and treatment of esophageal carcinoma, for the following purposes: (1) to present the standard practice for the diagnosis and treatment of esophageal carcinoma with a high regard for the principles of evidence-based medicine (EBM); (2) to improve the safety and results of

This article originally appeared in Japanese as Shokudo gan shindan · chiryo gaidorain (Guidelines for Diagnosis and Treatment of Carcinoma of the Esophagus), published by Kanehara, Tokyo, April.

This article does not contain any studies with human or animal subjects performed by any author(s). This paper consists of summary and text of Guidelines for Diagnosis and Treatment of Carcinoma of the Esophagus April 2012 edited without references and clinical questions and answers and grade of recommendation.

H. Kuwano $(\bowtie) \cdot$ T. Miyazaki

Department of General Surgical Science, Gunma University Graduate School of Medicine, 3-39-22 Showa-machi, Maebashi, Gunma 371-8511, Japan

e-mail: hkuwano@gunma-u.ac.jp

Y. Nishimura

Department of Radiation Oncology, Kinki University Faculty

of Medicine, Osaka-Sayama, Osaka, Japan

T. Oyama

Department of Gastroenterology, Saku Central Hospital,

Nagano, Japan treatment, thereby reducing the difference in treatment results among different institutions; (3) to reduce unnecessary costs and efforts; (4) help enable people to undergo treatment without anxiety.

These guidelines provide only guidance on the indications for treatment and do not restrict or prohibit the use of any treatment deviating from those described herein.

\section{Responsibilities}

The Japan Esophageal Society assumes responsibility for the content described in these guidelines.

However, responsibility for the treatment results should be borne by the doctor providing the treatment and shall not rest with the Japan Esophageal Society.

Basic principles adopted for the preparation of these guidelines

These guidelines only present indications for the treatment procedures and do not address the technical problems of each treatment modality. The principles of presenting adequate treatment procedures include the following:

\author{
H. Kato \\ First Department of Surgery, Dokkyo Medical University, \\ Tochigi, Japan \\ Y. Kitagawa \\ Department of Surgery, School of Medicine, Keio University, \\ Tokyo, Japan \\ M. Kusano \\ Department of Endoscopy and Endoscopic Surgery, \\ Gunma University Hospital, Maebashi, Gunma, Japan
}


(1) treatments are introduced in relation to the progression of carcinoma, without excess or deficiency; (2) the therapeutic efficacy is evaluated by evidence-based approaches; (3) the final evaluation of treatment procedures is based on the survival period, but remission of symptoms, reduction of tumor, and improvement of quality of life (QOL) are also taken into account; (4) evaluation is carried out depending on the location of the lesion. We propose to revise these guidelines continually along with advances in medical science.

Choice of treatment and patients' consent

When choosing a treatment, regardless of whether it is made based on these guidelines or not, it is necessary that the doctors explain the details of the treatment, the reasons for choosing it, possible complications, treatment results, etc., to patients to obtain the patients' understanding and informed consent.

\section{Level of recommendation}

"Clinical Questions" are attached to each item, and the levels of evidence/strength of recommendation for each item is indicated according to Minds (Medical Information Network Distribution System: http://minds.jcqhc.or.jp/n/) classification of recommendation grades (A, B, C1, C2, D), together with the recommendation grades of the Committee

H. Shimada

Department of Surgery, Tokai University Oiso Hospital, Oiso,

Kanagawa, Japan

H. Takiuchi

Cancer Chemotherapy Center, Osaka Medical College, Takatsuki, Osaka, Japan

Y. Toh

Department of Gastroenterological Surgery, National Kyushu

Cancer Center, Fukuoka, Japan

Y. Doki

Department of Gastroenterological Surgery, Osaka University

Graduate School of Medicine, Suita, Osaka, Japan

\section{Y. Naomoto}

Department of General Surgery, Kawasaki Medical School,

Okayama, Japan

H. Matsubara

Department of Frontier Surgery, Graduate School of Medicine, Chiba University, Chiba, Japan

M. Muto

Department of Gastroenterology and Hepatology, Kyoto

University, Kyoto, Japan

A. Yanagisawa

Department of Surgical Pathology, Graduate School of Medical

Science, Kyoto Prefectural University of Medicine, Kyoto, Japan to Develop Guidelines for Diagnosis and Treatment of Carcinoma of the Esophagus.

Algorithm for treatment of esophageal carcinoma (Fig. 1)

\section{Epidemiology, present status, and risk factors}

\section{Summary}

With regard to the dynamic trends of esophageal carcinoma in Japan, the incidence rate ${ }^{1}$ has been increasing slowly in men, whereas it has been leveling off in women. The mortality has been leveling off in men, but has been decreasing in women.

Currently, among patients with esophageal carcinoma, the percentage of males is higher, and the percentage of patients in their $60 \mathrm{~s}$ to $70 \mathrm{~s}$ is high. The carcinoma is most frequently located in the middle thoracic esophagus. Squamous cell carcinoma is the predominant histologic type. Esophageal carcinoma is frequently associated with synchronous or metachronous multiple carcinoma.

The cited risk factors include smoking and alcohol drinking in the case of squamous cell carcinoma. In regard to the risk factors for adenocarcinoma, Barrett's epithelium derived from persistent inflammation of the lower esophagus due to gastroesophageal reflux disease (GERD) has been reported to serve as the background mucosa for esophageal carcinoma in Europe and North America. In Japan, however, the risk associated with this factor remains unclear because of the scarcity of patients.

\section{Morbidity and mortality}

According to statistics issued by the Center for Cancer Control and Information, National Cancer Center based on cancer morbidity data derived from the Population-Based Cancer Registry, the estimated incidence rate in 2004 (crude incidence rate) was 24.4 persons per 100,000 population in men and 4.0 persons per 100,000 population in women. The age-adjusted incidence rate ${ }^{2}$ has been showing

\footnotetext{
${ }^{1}$ Incidence rate The number of cases detected in a certain population during a certain period of time divided by the number of individuals in the population. The data shown are those provided by the Center for Cancer Control and Information Services, National Cancer Center, on the basis of the national statistics of cancer morbidity data derived from the Population-Based Cancer Registry (1975-2005).

2 Age-adjusted incidence rate The incidence rate that would have been observed if the composition of the population was the same as the base population.
} 


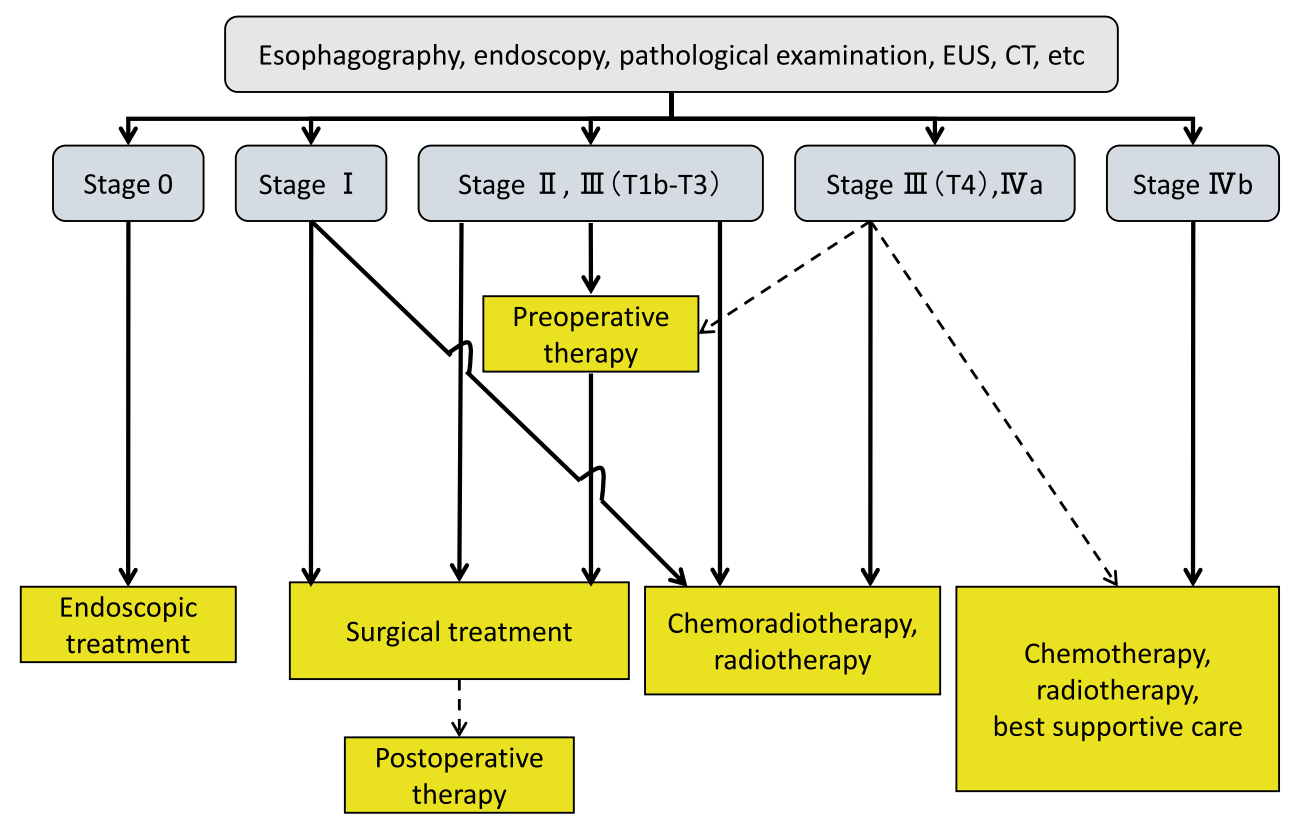

Stage is based on the 10th edition of the Japanese Classification of Esophageal Cancer.

Fig. 1 Algorithm for treatment of esophageal carcinoma

an upward trend in men, whereas there has been no particular pattern of increase or decrease recently in women.

According to the vital statistics compiled by the Ministry of Health, Labour and Welfare, there were 11,746 deaths from esophageal carcinoma in 2008 (the crude mortality rate ${ }^{3} 9.3$ persons per 100,000 ), which accounted for $3.4 \%$ of all deaths from malignant neoplasms. The crude mortality rate associated with esophageal carcinoma was 16.3 persons per 100,000 population in men, ranking after cancers of the lung, stomach, large intestine, liver, and pancreas. The corresponding rate was 2.7 persons per 100,000 population in women, ranking below the 10th place. The age-adjusted mortality rate ${ }^{4}$ of esophageal carcinoma has been leveling off in men and decreasing in women.

Cancer mortality data from vital statistics and various graphs based on this data are available at the Center

\footnotetext{
${ }^{3}$ Crude mortality rate: The number of deaths occurring during a certain period of time divided by the population during the period.

${ }^{4}$ Age-adjusted mortality rate The mortality rate that would have been observed if the composition of the population was the same as the base population. Because the cancer mortality rate increases as the age advances, the crude mortality rate increases in a population containing a greater proportion of elderly people than in one containing a smaller proportion of elderly people. Therefore, the mortality rate in a population as a whole is obtained in a way adjusted for the age composition of a population used as the base (base population). The 1985 model population (virtual population model based on Japan's population in 1985) is the base population used in Japan.
}

for Cancer Control and Information Services, National Cancer Center (http://ganjoho.jp/professional/statistics/ index.html).

\section{Present status of esophageal carcinoma in Japan}

With regard to the present status of esophageal carcinoma in Japan, a nationwide survey conducted by the Japan Esophageal Society (2002) revealed that male patients outnumbered female patients, with a malefemale ratio of about $6: 1$. Most patients were in their $60 \mathrm{~s}$ or $70 \mathrm{~s}$, accounting for about $68 \%$ of the patients overall. The carcinomas were predominantly located in the middle thoracic esophagus $(51.6 \%)$, followed in frequency by the lower thoracic esophagus (24.2\%), upper thoracic esophagus (13.4\%), abdominal esophagus $(4.5 \%)$, and cervical esophagus $(4.0 \%)$. Squamous cell carcinoma was the overwhelmingly frequent histologic type, accounting for $92.9 \%$ of all cases, followed in frequency by adenocarcinoma $(2.4 \%)$. A family history of malignant tumor was found in $22.7 \%$ of patients. A family history of esophageal carcinoma was present in $17 \%$ of all cases with a family history of malignant tumor, although gastric cancer was the most frequent at $28.9 \%$. About $20 \%$ of patients with esophageal carcinoma have synchronous or metachronous multiple cancer, which was the most frequently observed gastric cancer, followed by pharyngeal cancer, representing an important issue in the clinical diagnosis and treatment of 
esophageal carcinoma (see "Diagnosis and treatment of double carcinoma”).

\section{Risk factors}

The risk factors for esophageal carcinoma are alcohol drinking and smoking.

Alcohol and smoking are important risk factors for squamous cell carcinoma, serving as risk factors in more than $90 \%$ of all cases of esophageal carcinoma in Japan. It is known that the risk of developing esophageal carcinoma is increased by concomitant use of tobacco and alcohol. In October 2009, a working group of the World Health Organization prescribed that acetaldehyde associated with alcoholic beverages is a Group 1 carcinogen. In addition, in relation to dietary factors, poor nutritional status and vitamin deficiency due to inadequate intake of fruits and vegetables are also reported as risk factors. By contrast, intake of green and yellow vegetables and fruits are reported as preventive factors.

Although adenocarcinoma accounts for only a small percentage of patients with esophageal carcinoma, the percentage is increasing in Europe and North America, accounting for about more than half of all the cases of esophageal carcinoma. Barrett's epithelium caused by persistent inflammation of the lower esophagus due to gastroesophageal reflux disease (GERD) is known to serve as the background mucosa of the disease in Europe and America. GERD, high BMI, which serves as a risk factor for GERD, and smoking are involved in the development of the disease. In Japan, no clear evidence has been established because of the scarcity of cases.

\section{Diagnosis of esophageal carcinoma}

Diagnosis of the stage of carcinoma

\section{Summary}

The clinical stage of esophageal carcinoma is determined by various diagnostic imaging procedures in terms of the depth of tumor invasion, and presence/absence of lymph node metastasis and distant metastasis. Patients should be informed of the therapeutic strategies based on the assessment of the characteristic features of the lesion (grade of malignancy) and their general condition. Therapeutic strategies should be decided after explaining the basis for and process of diagnosis to patients, and obtaining their understanding, will, and consent (Fig. 2).

Evaluation of the general condition

Radical surgery for esophageal carcinoma, particularly surgery accompanied by thoracolaparotomy, is the most invasive among various types of surgery for gastrointestinal carcinomas. Recent advances in surgical techniques, anesthetic procedures, postoperative management, etc., have led it to safer and more radical treatment for esophageal carcinoma. However, the incidences of postoperative complications and surgery-related mortality still remain higher than those for other diseases. It should also be noted that esophageal carcinoma occurs predominantly in people of advanced age, i.e., 65-70 years of age, and that people of this age group are more likely to have various lifestylerelated diseases (hypertension, diabetes mellitus, hyperlipidemia, etc.). Therefore, it is desirable that application of
Fig. 2 Algorithm for deciding the therapeutic strategies for esophageal carcinoma

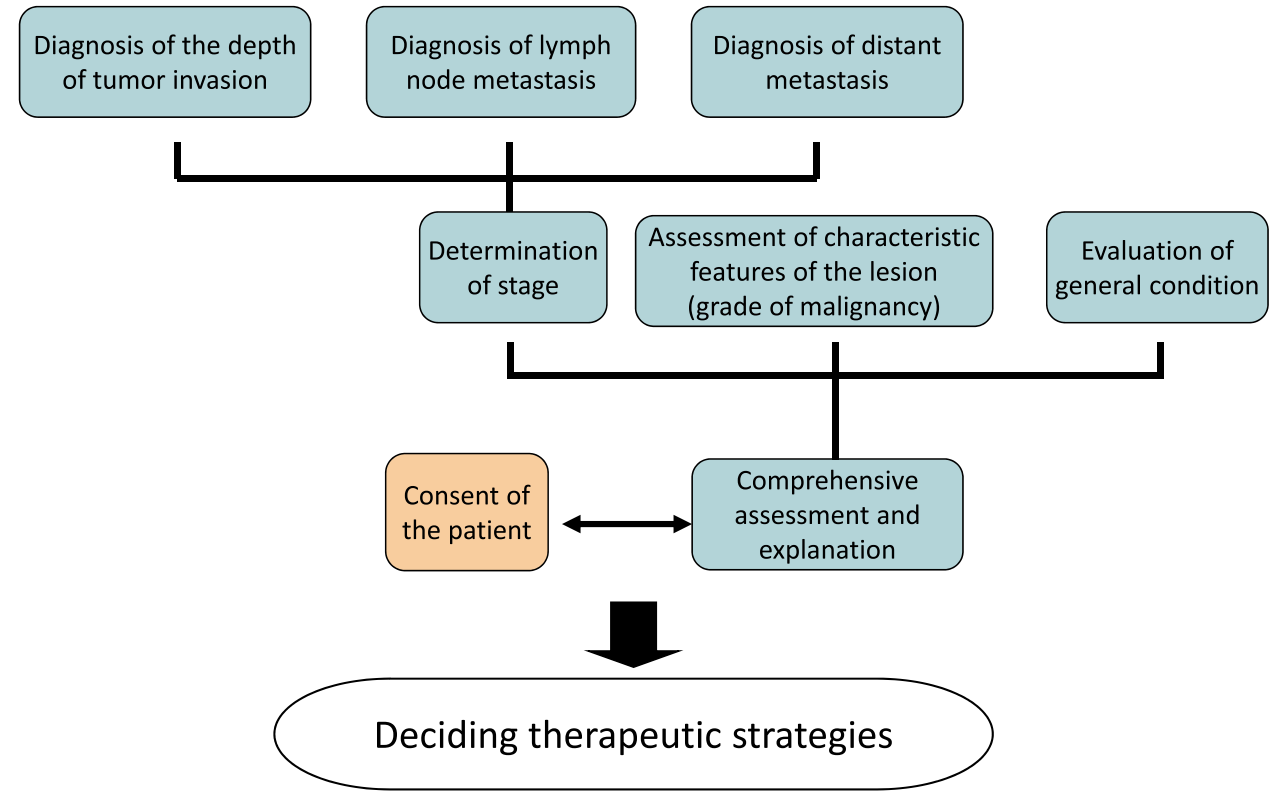


radical surgery be decided with due caution after evaluation of vital organ function. When applying chemotherapy, radiotherapy or chemoradiotherapy, it is desirable that the functions of vital organs meet certain criteria for implementation of the therapy.

In this connection, several tests that are required for evaluating the general condition and function of major organs are described below, in addition to rough guides for judging the test results. However, therapy based on the patient's general condition should follow comprehensive evaluation and it is not easy to establish accurate numerical standards.

\section{Performance status (PS)}

Performance status is a simple and useful index commonly used for comprehensive evaluation of the patient's general condition (Table 1). Esophageal carcinoma patients with PS scores of $0-2$, as described below, are generally considered as suitable candidates for radical surgery, chemotherapy, or radiotherapy.

\section{Pulmonary function tests}

Aging and smoking history are risk factors in patients with carcinoma of the esophagus, and the prevalence of chronic obstructive lung disease is relatively high among these patients. Pulmonary function test results are important indices for deciding the advisability of thoracotomy. Whether or not thoracotomy is indicated should be judged comprehensively, taking into account the results of spirometry (\%VC, FEV1.0 \%, \%RV/TLC), arterial blood gas analysis, chest radiography findings, CT findings, smoking history, and past medical history. Thoracotomy should be considered carefully if the values of $\% \mathrm{VC}$ is $40 \%$ or less, FEV1.0 \% is $50 \%$ or less, FEV1.0 is less than $1.5 \mathrm{~L}$, and \%RV/TLC is $56 \%$ or more, respectively, and the arterial oxygen tension is 60 Torr or less.

Table 1 Eastern Cooperative Oncology Group (ECOG) activity score

PS $0 \quad$ Fully active and able to carry out all pre-disease activities without restriction

PS 1 Restricted in physically strenuous activities, but ambulatory and able to carry out work of a light or sedentary nature, e.g., light housework and office work

PS 2 Ambulatory and capable of all self-care, but unable to carry out any work activities. Up and about more than $50 \%$ of waking hours

PS 3 Capable of only limited self-care, confined to bed or chair more than $50 \%$ of waking hours

PS 4 Completely disabled. Cannot carry on any self-care. Totally confined to bed or chair

\section{Cardiac function tests}

In principle, surgery is not indicated in patients with heart failure due to valvular disease or cardiomyopathy, severe arrhythmia, or myocardial infarction within 3 months prior to the onset. A resting or exercise electrocardiography (ECG) is carried out as a rule. If any abnormality is found, the patient should be subjected to Holter ECG monitoring, echocardiography, cardiac catheterization, or exercise stress perfusion imaging.

It is desirable to consult with a cardiovascular internist if the patient has cardiac function abnormalities or has been on antiplatelet therapy or anticoagulant therapy before surgery.

\section{Hepatic function tests}

Surgical treatment is basically not indicated for severe hepatitis or fulminant hepatitis. In cases of chronic hepatitis or hepatic cirrhosis, surgical treatment should be considered based on a comprehensive evaluation of blood counts and the results of blood coagulation tests, blood biochemistry tests, ICG stress test $(15 \mathrm{~min})$, and hepatitis screening. Excluding special conditions, surgery is basically not performed in patients who have an ICG retention rate of $40 \%$ or more at $15 \mathrm{~min}$ due to hepatic dysfunction. If the value is $20-40 \%$, application of minimally invasive surgery including reduction of operative radicality may be considered with due caution.

It has been pointed out that systemic chemotherapy may cause reactivation of hepatitis B virus in HBs antigenpositive patients. Chemotherapy may also cause fulminant hepatitis in patients who have a history of hepatitis B virus infection (negative for $\mathrm{HBs}$ antigen and positive for $\mathrm{HBc}$ antibody or HBs antibody). Therefore, it is desirable to consult a hepatologist beforehand.

\section{Renal function tests}

Evaluation of renal function includes general urinalysis, serum creatinine, BUN, electrolytes, and creatinine clearance (Ccr). Although it is relatively rare for surgery to be ruled out only because of renal function deterioration, it is desirable to explain to the patient the possible need for dialysis therapy if the serum $\mathrm{Cr}$ level is $2.0 \mathrm{mg} / \mathrm{dL}$ or more and the Ccr is $30 \%$ or less.

\section{Glucose tolerance test}

Perioperative blood glucose control should be implemented strictly in patients with diabetes or decreased glucose tolerance. Measurement of fasting blood glucose levels, the oral 


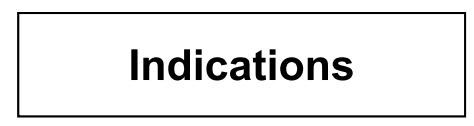

- EP or LPM lesions

\section{Relative indications}

- MM or SM1 lesions not accompanied by clinical evidence of lymph node metastasis

\section{Investigational stage}

- SM2 or deeper lesions targeted for local control

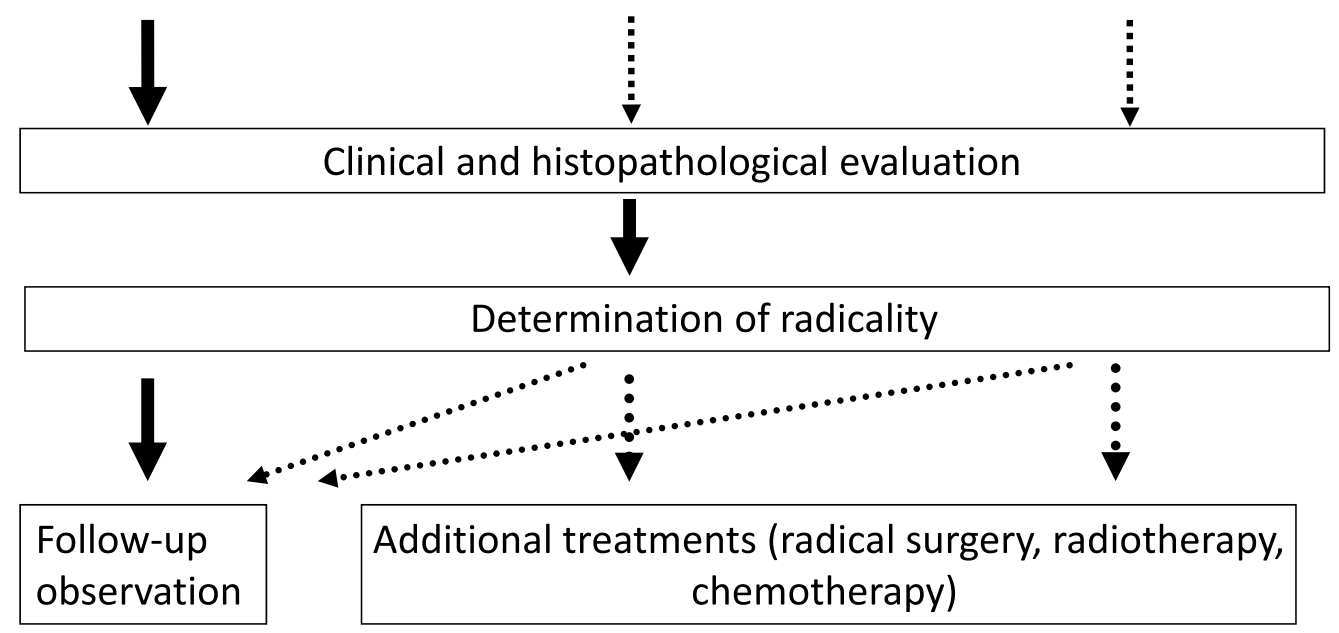

Fig. 3 Indications for endoscopic resection

$75 \mathrm{~g}$ glucose tolerance test, measurement of HbA1c levels, quantitative urinary glucose measurement, and urinary ketone testing should be performed. Preoperative control is targeted at a fasting blood glucose of $<140 \mathrm{mg} / \mathrm{dl}$, daily urinary glucose excretion of $\leq 10 \mathrm{~g}$, and negative test for urinary ketones.

\section{Other considerations}

Central nervous system function, including the presence/ absence of mental disorders, should be evaluated comprehensively. In general, radical surgery is not indicated for patients with carcinoma of the esophagus when there is a concomitant acute-phase cerebrovascular disorder. It is desirable that patients with depression, anxiety, delirium, or dementia be referred for professional evaluation by a psychiatrist.

\section{Endoscopic treatment}

Summary

The mainstay of endoscopic treatment is endoscopic resection (ER).

Endoscopic resection includes conventional endoscopic mucosal resection (EMR), in which the affected mucosa is held or aspirated and resected with a snare, and endoscopic submucosal dissection (ESD), which refers to en bloc resection of an extensive lesion using an IT knife or a hook knife. Other available endoscopic treatments include photodynamic therapy (PDT), ${ }^{5}$ argon plasma coagulation therapy, ${ }^{6}$ and electromagnetic coagulation therapy.

Indications for endoscopic resection (Fig. 3) Among lesions that do not exceed the mucosal layer (T1a), those remaining within the mucosal epithelium (EP) or the lamina propria mucosae (LPM) are extremely rarely associated with lymph node metastasis; therefore, endoscopic resection is a sufficiently radical treatment for these lesions. Lesions reaching the muscularis mucosae or slightly infiltrating the submucosa (up to $200 \mu \mathrm{m}, \mathrm{T} 1 \mathrm{~b}-\mathrm{SM} 1$ ) are amenable to mucosal resection, but may have a risk of lymph node metastasis. Therefore, these cases represent relative indications. Furthermore, $50 \%$ of lesions invading deeper (more than $200 \mu \mathrm{m}$ ) into the submucosa (T1b-SM2) are associated with metastasis, and even superficial carcinomas

\footnotetext{
5 Photodynamic therapy (PDT) In this treatment modality, the oncotropic, light-sensitive substance porfimer sodium (Photofrin) is injected intravenously, and tumor tissue is necrotized by applying $630 \mathrm{~nm}$ red light to the light-sensitive substance selectively incorporated into the tumor. Use of this treatment for early carcinomas of the lung, stomach, and uterus and superficial esophageal carcinoma has been covered by the national health insurance in Japan since October 1994. The reported local control rate in cases of superficial esophageal carcinoma is $90 \%$. PDT is reported to be effective for unresectable cases by ER and remnant lesions after ER, radiation therapy, or chemoradiation therapy.

${ }^{6}$ Argon plasma coagulation (APC) This is an endoscopic treatment method in which argon gas is applied, and tissue is cauterized by thermocoagulation using high-frequency currents.
} 
should be treated in the same manner as advanced carcinomas (carcinomas exceeding the muscularis propria).

Mucosal resection covering 3/4 of the entire circumference is likely to be associated with postoperative cicatricial stenosis. Therefore, sufficient explanation should be given to the patient prior to the operation and preventive measures must be taken. In cases of superficially spreading carcinoma, deep infiltration may occur in several areas, necessitating careful diagnosis of the depth of invasion.

Diagnosis of resected tissue specimens There are limitations to all modes of diagnosis of the depth of tumor invasion. It is also difficult to accurately determine the depth of invasion of extensive lesions. Thus, tissue specimens obtained by en bloc resection are necessary.

Treatment of lesions not amenable to ER Elevation of the mucosa may pose difficulty in additional ER of lesions remaining marginally after ER or ER after radiotherapy or chemoradiotherapy. These cases and cases with a bleeding tendency are not amenable to ER, and consideration of other treatment options such as photodynamic therapy (PDT) and argon plasma coagulation (APC) is required.

Superiority of en bloc resection En bloc resection is desirable for histologic diagnosis of resected specimens. Endoscopic submucosal dissection (ESD) enables en bloc resection of lesions which formerly were subjected to fractional resection. Further development of equipment and spread of improved techniques are anticipated.

Complications Various complications, including bleeding, esophageal perforation, and cicatricial stenosis have been reported in association with ER, including ESD. The need for prevention, prophylactic measures, and treatment of these complications should be well recognized. There has been extensive discussion on the need for additional treatment after diagnosis of resected tissue.

\section{Indications for endoscopic resection}

\section{Handling of resected specimens and evaluation of the integrity of treatment}

The rules for the handling of endoscopically resected specimens and the procedures for histopathological examination to help determine therapeutic strategies are as follows:

1. Only specimens obtained by en bloc resection should be used for histopathological evaluation of the adequacy of resection.

2. The following procedures should be undertaken when handling specimens, in accordance with the Guidelines for Clinical and Pathologic Studies on Carcinoma of the Esophagus, 10th edition.
- $\quad$ EMR and ESD specimens should be stretched out so that they are approximately the same size as in vivo, and the proximal and distal ends should be identified. Specimens should then be promptly fixed.

- Iodine staining is recommended before sectioning, to identify the iodine-unstained area.

- Specimens should be sectioned in a direction that allows accurate assessment of the resection margins. When the specimen has adequate resection margins, it should be sectioned transversely (perpendicular to the long axis) to obtain as much information as possible. When the specimen has small resection margins, it should be sectioned perpendicular to the tangent line at the smallest margin. The entire specimen should be continuously sectioned at $2-3 \mathrm{~mm}$ intervals. The cut surface should include the full thickness of the epithelium and the muscularis mucosae (Fig. 4).

3. The histopathological diagnosis should be made by examination of all the sections obtained by the above process.

4. The objective of performing histopathological diagnosis of endoscopically resected specimens is to determine whether any additional treatment is necessary. The depth of invasion, presence/absence of lymphovascular invasion, and status of the resection margin should therefore be specified to determine whether the lesion has been completely removed and to assess the likelihood of metastasis.

\section{Surgical treatment}

\section{Summary}

Therapeutic strategies vary according to factors such as the location of the esophageal lesion, extent of the lesion, depth of invasion, presence/absence of metastasis, general condition of the patient, and the institution that provides the treatment. There are many treatment options available. Some treatments are already formulated and used commonly in daily clinical practice. Some other treatments are in the clinical research phase and in the process of expanded use, although lacking in solid supportive evidence.

With regard to surgical treatment, there are various options depending on the institution as to the width of the resection margin, extent of lymph node dissection, the organ and route used for reconstruction, multimodality therapy including adjuvant therapy, and salvage surgery following definitive (chemo-) radiotherapy. Therefore, it is difficult to choose the currently most appropriate standard therapy based on evidence.

The esophagus extends anatomically from the cervical through the thoracic region into the abdominal region. It 
1. When the specimen has adequate resection margins, it should be sectioned transversely.

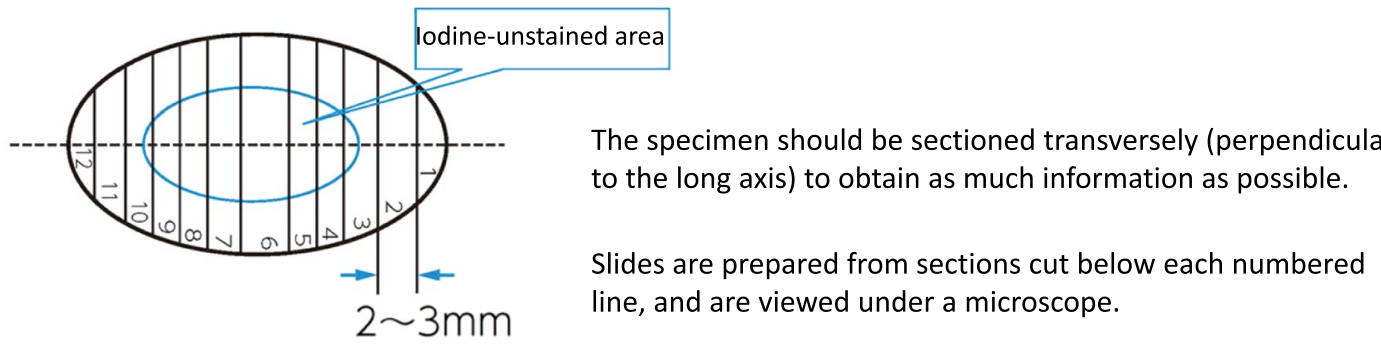

2. When the resection margin is small, the specimen should be sectioned perpendicular to the tangent line at the closest margin.

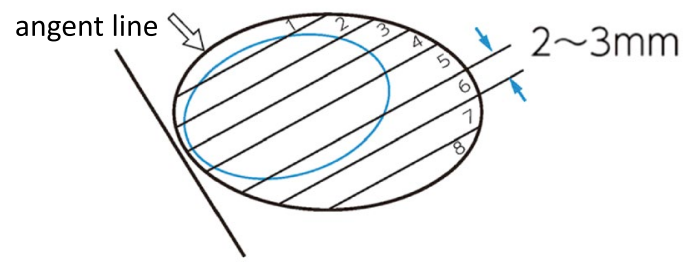

Slides are prepared from sections cut below each numbered line, and are viewed under a microscope.

When section No. 1 includes part of the lesion, it should be inverted $(\Rightarrow)$ so that slides are prepared from above the numbered line to evaluate the edge of the lesion.

Fig. 4 Sectioning of specimens obtained by endoscopic resection. The specimen should be cut after the iodine-unstained area is identified by the staining

is surrounded by various organs according to its location. Although therapeutic strategies vary widely according to the location of the tumor, stage, and general condition of the patient, surgery remains the mainstay of treatment. In general, absolute indications for endoscopic treatment are carcinomas with a depth of invasion classified as EP or LPM. However, esophagectomy and reconstruction not accompanied by lymph node dissection may be indicated as a comprehensive judgment for extensive early carcinomas without clinical lymph node metastasis. For lesions reaching up to the muscularis mucosa, the frequency of lymph node metastasis is about $9.3 \%$. This percentage increases as the depth of invasion increases. For lesions invading deeper into the submucosal tissue, the rate of metastasis is about $50 \%$. In general, if there is reasonable ground for suspecting lymph node metastasis, lymph node dissection should be carried out even for superficial lesions in the treatment of T2 or deeper carcinomas. Surgery may be considered in patients with T4 lesions, only when curative resection is judged to be applicable. Basically, surgery is not chosen as the initial treatment when there is metastasis to distant lymph nodes or other organs.

At present, efforts are focused on the establishment of surgical treatments by the formulation and standardization of surgical techniques represented by three-field lymph node dissection (cervical, thoracic, and abdominal) for cases of thoracic esophageal carcinoma, as well as the introduction and promotion of endoscopic surgery (videoassisted surgery) based on established surgical treatments and minimization of surgical invasion through the use of limited surgery. However, supportive evidence to promote these actions still remains inadequate.

\section{Surgery for cervical esophageal carcinoma}

Summary The anatomical structure and physiological functions of the hypopharynx to the cervical esophagus are complicated. Because the loss of vocal function by combined laryngectomy greatly affects the postoperative QOL of the patient, the surgical procedure should be determined carefully, after due consideration given to the balance between the radicality of the treatment and the QOL.

\section{Resection}

Carcinoma of the cervical esophagus is often advanced at diagnosis, with a high incidence of lymph node metastasis and infiltration into other organs. However, lymph node metastasis is mainly restricted to the cervical region, and radical surgery is often applicable. Patients who have extensive distant metastases and metastasis to a number of superior mediastinal lymph nodes are usually not suitable candidates for radical resection.

Larynx-conserving surgery

This operation is indicated for patients in whom the tumor has not invaded the larynx or trachea and in whom the proximal end of the tumor remains inferior to the esophageal orifice.

Larynx-conserving surgery is divided into larynx-conserving cervical esophagectomy and larynx-conserving total esophagectomy according to the necessity for thoracic esophagectomy. Concomitant resection of the thoracic 
esophagus may be performed when the tumor has invaded the thoracic esophagus, when there are multiple lesions not amenable to endoscopic resection, or when it is difficult to perform reconstruction requiring microvascular anastomosis. This surgery may be indicated for patients in whom preoperative therapy results in tumor shrinkage.

Laryngopharyngoesophagectomy (combined laryngectomy)

Laryngopharyngoesophagectomy is indicated for patients with tumors invading the larynx, trachea, and hypopharynx, or those in whom sufficient preservation of the cervical esophagus to perform anastomosis is difficult.

This type of surgery is divided into laryngopharyngectomy plus cervical esophagectomy and laryngopharyngectomy plus total esophagectomy according to the necessity for thoracic esophagectomy. Resection of the thoracic esophagus may be combined when the tumor has invaded the thoracic esophagus, when there are multiple lesions not suitable for endoscopic resection, or when it is difficult to perform reconstruction requiring microvascular anastomosis.

\section{Lymph node dissection}

According to the 10th edition of the Japanese Classification of Esophageal Cancer, cervical lymph nodes include superficial cervical nodes of the neck [100], cervical paraesophageal lymph nodes [101], deep cervical lymph nodes [102], peripharyngeal lymph nodes [103], and supraclavicular lymph nodes [104]. The major thoracic lymph nodes are the recurrent laryngeal nerve lymph nodes [106-rec] and upper thoracic paraesophageal lymph nodes [105].

Among these, [101] and [106-rec] lymph nodes belong to group 1, and [102], [104] and [105] lymph nodes belong to group 2 in cases of cervical esophageal carcinoma. It is preferable to remove them.

\section{Method of reconstruction}

Although reconstruction using a free intestinal graft is common when the operation involves cervical manipulation alone, gastric tube reconstruction may be employed in some cases. Myocutaneous flaps or skin rolls may also be used for reconstruction. When concomitant thoracic esophagectomy is performed, the stomach or colon is used for reconstruction as in cases of usual reconstruction following resection of the thoracic esophageal carcinoma. However, a free jejunal graft may be added if the length of the organ prepared for reconstruction is not sufficient.

\section{Surgery for thoracic esophageal carcinoma}

Summary Thoracic esophageal carcinoma is often accompanied by extensive lymph node metastasis in the cervical, thoracic, and abdominal regions. A right thoracotomy with total extirpation of the thoracoabdominal esophagus and lymph node dissection is generally carried out. The width of the resection margin of the tumor should be decided bearing in mind both the possibility of persistent carcinoma in the remaining esophageal wall and the extent of lymph node dissection.

In cases of thoracic esophageal carcinoma, the extent of lymph node dissection should be determined after preoperative evaluation of the location, size, and depth of invasion of the carcinoma by imaging modalities including computed tomography (CT), magnetic resonance imaging (MRI), and PET.

Three routes of reconstruction, i.e., antethoracic, retrosternal, and posterior mediastinal are available. Although each of these routes has its own advantages and disadvantages, the posterior mediastinal route has been most frequently employed recently. The stomach is the most common organ used for reconstruction.

\section{Resection}

Thoracic esophageal carcinoma is often accompanied by extensive lymph node metastasis in the cervical, thoracic, and abdominal regions. A right thoracotomy with total extirpation of the thoracoabdominal esophagus, and lymph node dissection of the lymph nodes in all the three regions (cervical, thoracic, and abdominal) is generally carried out.

The width of the margin of resection of the tumor should be decided bearing in mind both the possibility of persistent carcinoma in the remaining esophageal wall and the extent of lymph node dissection. Persistent carcinoma in the esophageal wall may result from intramural spread, intraepithelial spread, vascular invasion, or intramural metastasis. Submucosal spread increases according to the tumor depth, reportedly reaching $30 \mathrm{~mm}$ in cases of $\mathrm{T} 2$ carcinoma. There is no standard extent of resection established for all of the above cases, and a sufficient margin may not be available at the proximal end of the resection according to the location of the tumor. Thus, the extent of resection should be decided on the basis of the findings on preoperative esophagography and endoscopy, intraoperative rapid frozen-section diagnosis, and intraoperative macroscopic evaluation including close observation of the mucosal surface (and iodine application) under intraoperative esophagotomy.

\section{Endoscopic esophagectomy and reconstruction}

Thoracoscopy- or laparoscopy-assisted esophagectomy with esophageal reconstruction and mediastinoscopy- or laparoscopy-assisted transhiatal esophagectomy have been reported as promising surgical procedures, although they are still in the investigational stages, in view of the minimal invasiveness, radicality, and the long-term results. In Japan, endoscopic esophagectomy is almost always performed as a radical surgery which includes sufficient lymph node dissection comparable to open surgery. Indications for this operation vary among institutions and it is used for T3 cancer in some institutions. It has been reported that endoscopic esophagectomy is comparable to conventional standard surgery with open thoracotomy 
in terms of the operating time, amount of blood loss, and number of dissected lymph nodes, and is advantageous in terms of providing early relief from postoperative pain and rapid restoration of vital capacity, as long as it is carried out at institutions with accumulated clinical experience. On the negative side, recurrent laryngeal nerve paralysis is reported to occur more frequently after this procedure than after standard surgery via thoracotomy.

Some techniques have been suggested for performing carrying out implementing safe endoscopic surgery with reduced operating time and improved accuracy of node dissection. These techniques include for direct manipulations, via a minor thoracotomy, video-assisted thoracoscopic surgery (VATS) with minor thoracotomy and hand-assisted laparoscopic surgery (HALS), involving manipulation with one hand on the abdomen. Although thoracic manipulations have been predominantly carried out with the patient in the left lateral decubitus position, complete endoscopic thoracic manipulations with the patient in the prone position is becoming more and more frequent recently. Transhiatal esophagectomy with mediastinal dissection using a mediastinoscope inserted via a cervical incision or with mediastinal dissection via laparotomy has also been proposed. It is reported that endoscopic surgery allows node dissection with improved accuracy because of the higher-power visualization that allows observation of microanatomy. However, no definitive conclusions have been reached yet as to the longterm outcomes of this form of surgery as compared to those of conventional standard surgery with open thoracotomy and node dissection, and further investigation in randomized controlled trials is awaited.

\section{Lymph node dissection}

Thoracic esophageal carcinoma is commonly accompanied by lymph node metastasis in extensive areas from the cervical to the abdominal region. However, because the distribution and incidence of lymph node metastasis vary according to the location, size, and depth of invasion of the tumor, preoperative evaluation of individual patients by CT, US, MRI, and PET is important to determine the extent of lymph node dissection.

Radical surgery for thoracic esophageal carcinoma is accomplished ordinarily as a combination of three approaches, i.e., the cervical, thoracic, or abdominal approaches. The mediastinal approach has also been proposed as an alternative to the cervical approach for dissection of the cervical paraesophageal lymph nodes [101].

Upper thoracic esophageal carcinoma $(U t)$

Patients with this type of lesion usually present with lymph node metastasis, mainly in the cervical to upper mediastinal region; thus, lymph node dissection should include the cervical region. Addition of median sternotomy or manubriotomy has also been suggested to allow a better field of view of the cervicothoracic junction region. Although metastasis to the lower mediastinal or abdominal lymph nodes occurs less frequently in cases of Ut, dissection should ordinarily cover all the three regions, i.e., cervical, thoracic, and abdominal regions, including the left gastric artery lymph nodes.

\section{Middle thoracic esophageal carcinoma (Mt)}

In general, metastatic lymph nodes in cases of Mt are relatively evenly distributed over the cervical to upper, middle, and lower mediastinal and abdominal regions. Because the involvement of cervical lymph nodes other than the cervical paraesophageal lymph nodes [101] is relatively rare, lymph node dissection via the intrathoracic approach instead of the cervical approach has also been proposed.

When the thoracic approach is judged to be inadequate based on the preoperative diagnosis of metastasis, it is important to add a cervical approach to dissect the lymph nodes surrounding the bilateral recurrent laryngeal nerve up to the inferior pole of the thyroid. In particular, the lymph nodes of $101 \mathrm{~L}$ are difficult to be dissected thoroughly by thoracic manipulations alone, and additional dissection via a cervical incision is necessary. In addition, supraclavicular lymph nodes [104] cannot be dissected by thoracic manipulations, and a cervical approach is necessary for secure lymph node dissection in this region.

\section{Lower thoracic esophageal carcinoma $(L t)$}

In cases of Lt, while lymph node metastasis mainly occurs in the mediastinal and abdominal regions, metastasis to the cervical lymph nodes may also occur, albeit at a lower frequency. The optimal approach for lymph node dissection remains under discussion; while some propose adding the cervical approach, similar to the case for $\mathrm{Mt}$, others regard the thoracic approach as being superior. Because metastasis to the upper mediastinal lymph nodes is less frequent in cases of superficial carcinoma of the lower thoracic esophagus, there is a view that the extent of lymph node dissection could be minimized and that cervical lymph node dissection could be omitted altogether in some cases.

\section{Method of reconstruction (Table 2)}

\section{Route of reconstruction}

Three routes, i.e., the antethoracic, retrosternal, and posterior mediastinal (including intrathoracic) routes, are available for reconstruction. The route employed varies according to the case, and each route has its own advantages and disadvantages. Recently, the posterior mediastinal route is the most frequently used for reconstruction when high intrathoracic anastomosis is included. Because surgery for second primary cancer in the gastric tube is difficult after reconstruction via the posterior mediastinal route, the risk of carcinoma occurring in the gastric tube should be considered particularly when long survival is expected. 


\section{Organs used for reconstruction}

Reconstruction using the stomach is the most common method. In patients with past history of gastrectomy, those with a concomitant gastric carcinoma, and those in whom the stomach needs to be preserved, colic and ileocolic or jejunal grafts may be used.

\section{Anastomosis}

Anastomosis may be divided into cervical and intrathoracic depending on the site of anastomosis. This site of anastomosis is chosen according to the location of the tumor, the organ used for reconstruction, and the route of reconstruction. Intrathoracic anastomosis is associated with a high risk of serious complications in the event of anastomotic leakage. The anastomosis techniques include manual suture and mechanical suture. For intrathoracic anastomosis, mechanical suture using a circular stapler is frequently employed. Because anastomotic leakage and stricture exert a strong influence on the postoperative course and QOL of the patient, it is important to use appropriate anastomotic techniques tailored to individual patients.

\section{Surgery for carcinoma of the esophagogastric junction (abdominal esophageal carcinoma)}

Summary Similar to the case for surgical treatment of thoracic esophageal carcinoma, various techniques are available for the surgical treatment of carcinoma of the esophagogastric junction (E, EG). These include a right thoracotomy with dissection including the upper mediastinal lymph nodes and reconstruction using a gastric tube, lower esophagectomy with proximal gastrectomy or lower esophagectomy with total gastrectomy via left thoracolaparotomy or serial left thoracic and abdominal incisions, and a transhiatal approach to the lower mediastinum without thoracotomy. Metastasis involving the lower paraesophageal to upper abdominal lymph nodes is frequent in these cases. The most commonly employed technique is intrathoracic anastomosis using a gastric tube or the jejunum.

\section{Resection and lymph node dissection}

The 10th edition of the Japanese Classification of Esophageal Cancer defines the esophagogastric junction region as the region within $2 \mathrm{~cm}$ above and below the esophagogastric junction, and esophagogastric junction carcinoma as that with its center located within this region. According to this definition, abdominal esophageal carcinoma is included in this category. In cases of esophagogastric junction carcinoma extending more to the esophageal side than to the gastric side (E, EG), right thoracotomy with dissection including the upper mediastinal lymph nodes and reconstruction using a gastric tube are frequently performed in the same manner as for cases of thoracic esophageal carcinoma. In some cases, lower esophagectomy with proximal gastrectomy or lower esophagectomy with total 
gastrectomy via left thoracolaparotomy or serial left thoracic and abdominal incisions may be carried out, considering that cervical or upper mediastinal lymph node dissection is of lesser significance. A transabdominal approach to the lower mediastinum via dilated esophageal hiatus without thoracotomy is also reported. Metastasis to the lower thoracic paraesophageal lymph nodes [110], cardiac lymph nodes [1] [2], lesser curvature lymph nodes [3], left gastric artery lymph nodes [7], and celiac artery lymph nodes [9] is frequent.

In cases of esophagogastric junction carcinoma extending more to the gastric side than to the esophageal side ( $\mathrm{G}, \mathrm{GE})$, metastasis to the mediastinal lymph nodes is less frequent; thus dissection of these lymph nodes is of lesser consequence. Therefore, these lymph nodes are classified as group 3 in the 10th edition of the Japanese Classification of Esophageal Cancer.

\section{Method of reconstruction}

Intrathoracic anastomosis using a gastric tube, jejunal interposition by elevation of the jejunum, and intrathoracic anastomosis by the Roux-en-Y style are available. In cases of esophagogastric anastomosis following lower esophagectomy with proximal gastrectomy, postoperative reflux esophagitis is a potential problem and may require prophylactic measures.

\section{Other surgical treatments}

Summary Although radical surgery for esophageal carcinoma basically consists of resection, lymph node dissection, and reconstruction, other treatments may be carried out if it is difficult or unnecessary to complete these procedures because of various factors such as the stage and malignancy grade of the carcinoma and the general condition of the patient. Transhiatal esophagectomy has been performed as a radical operation for mucosal carcinoma or abdominal esophageal carcinoma that is difficult to treat with an endoscopic procedure and that presumably requires no mediastinal lymph node dissection. This has also been used as a palliative operation for patients who are not suitable candidates for thoracotomy and mediastinal lymph node dissection because of poor pulmonary function or other reasons. However, indications for this procedure have recently become limited because of the expanding application of endoscopic treatment as esophagus-preserving therapy and the spread of multidisciplinary therapy including chemoradiotherapy.

Bypass surgery may be performed as a palliative treatment for patients who have difficulty in oral intake because of esophageal stenosis due to esophageal carcinoma or changes after treatment for esophageal carcinoma. However, the indications for bypass surgery have become limited because of the spread of esophageal stenting.

\section{Transhiatal esophagectomy}

In this method, the thoracic esophagus is detached and removed via the cervical and abdominal approaches without thoracotomy. This technique was first reported by Turner in 1933. Akiyama et al. introduced this method in Japan in 1971, and it has since come to be used widely in clinical practice in this country. This technique has been employed for resection and reconstruction in patients with cervical esophageal carcinoma, patients with thoracic or abdominal esophageal carcinoma who are not suitable candidates for thoracotomy because of severe pleural adhesions or poor pulmonary function, patients of advanced age, and patients with mucosal carcinoma not requiring lymph node dissection. Although this technique allows dissection of the abdominal to lower mediastinal lymph nodes to some extent, upper to middle mediastinal dissection is difficult.

Currently, the use of transhiatal esophagectomy has become rare because of the spread of chemoradiotherapy and endoscopic submucosal dissection.

Treatments for unresectable cases

Advanced esophageal carcinomas that are unresectable because of infiltration into other organs or distant metastasis are initially treated by radiotherapy, chemotherapy, or chemoradiotherapy. However, patients with esophageal stenosis or trachea-esophageal fistula not responding to these treatments suffer from difficulty in oral intake, resulting in a marked decrease in QOL. Bypass surgery is a palliative treatment used to achieve oral feeding in these cases of malignant esophageal stenosis or obstruction. Currently, indications for bypass surgery have become rare because of the spread of covered esophageal stent placement.

In bypass surgery, the thoracic esophagus is excluded from the continuity of the digestive tract and a new route of oral intake is made. The route of bypass is antethoracic or retrosternal. Because most patients have advanced carcinoma and a poor nutritional status, anastomotic leakage occurs frequently, necessitating caution. In recent years, this procedure has been also employed as a palliative operation in patients in whom major lesions are judged to be unresectable during salvage surgery following definitive chemoradiotherapy.

\section{Perioperative management and clinical path}

Summary It was previously considered that introduction of the clinical path method would be difficult for perioperative management of esophageal carcinoma, because of the pathological features of this carcinoma and the diversity of surgical techniques available. In recent years, however, a clinical path for resection and reconstruction of the esophagus has been proposed by various institutions and has been applied in clinical practice. However, there has 
been only limited data from large-scale clinical studies evaluating the usefulness of a clinical path for perioperative management.

In recent years, an increasing number of institutions have included nutritional support teams (NST) for perioperative nutritional management of patients with esophageal carcinoma, facilitating early implementation of enteral nutrition. As an element of perioperative management, steroid administration is useful and recommended in postoperative management. Abstinence from smoking, respiratory physical therapy, and preoperative oral care are generally considered to be important for the prevention of postoperative complications.

Introduction of a clinical path in perioperative management of esophageal carcinoma

Clinical path is a treatment plan prepared to facilitate provision of safe team-approached medical care involving health-care professionals from multiple medical fields, including doctors, nurses, pharmacists, nutritionists, and physical therapists, with the aim of improving the quality of care through standardization of health care. In the USA, along with the introduction of the diagnosisrelated group/prospective payment system (DRG/PPS) in 1983, the fixed charge system, replacing the fee-forservice system, came to be employed for inpatient hospital care and a clinical path was introduced mainly for the purpose of reducing the length of stay in the hospital of patients and to reduce health-care costs. In Japan, around the time of the introduction of the diagnosis procedure combination (DPC) system, clinical paths were introduced for the treatment of various diseases in the 1990s. The use of clinical paths has recently come to be considered to be important not only for improvement of the quality of health care and medical education to provide safe team health care, but also for enhancement of patient-centered health care, including promotion of obtainment of informed consent.

In patients with esophageal carcinoma, the general condition is greatly affected by the disease state and surgery. Perioperative management techniques in these patients, including the treatment of concomitant diseases, are diverse, with large differences among institutions. Therefore, preparation of a simple clinical path has been considered to be difficult, as reflected by the scarcity of reports documenting the clinical usefulness of a clinical path. The various clinical paths for esophageal carcinoma reported to date are those developed by institutions to achieve safe perioperative management of patients undergoing resection and reconstruction of the esophagus. It is currently difficult to find evidence comparing the usefulness of clinical paths.

Clinical paths for diagnosis and treatment involving EMR and ESD, endoscopic treatment procedures for early esophageal carcinoma have already been developed and are in clinical use in many institutions. Clinical paths have also been introduced for thoracoscopy- or laparoscopy-assisted esophagectomy with reconstruction, which are used in high-volume centers treating a large number of patients.

Perioperative nutritional management of esophageal carcinoma

Nishi et al. have reported that spontaneous healing of anastomotic leakage after surgery for esophageal carcinoma requires a caloric intake of at least $45 \mathrm{kcal} / \mathrm{kg} /$ day, and that the serum albumin concentration must be maintained at $3.5 \mathrm{~g} / \mathrm{dL}$ or more. It has been reported that enteral nutrition in the perioperative period is associated with a lower incidence of perioperative complications, due to reduced production of endotoxins and inflammatory cytokines, as compared to parenteral nutrition. In cases of radical surgery for esophageal carcinoma, perioperative management by aggressive use of enteral nutrition has been reported to be helpful.

In patients undergoing radical surgery for esophageal carcinoma, the digestive and absorptive capability of the small intestine is often maintained in a normal condition. Therefore, it has been considered that early enteral nutrition rather than central venous nutrition is desirable to enhance postoperative immunity, etc. An enteral feeding tube should be placed during surgery, and a liquid diet should be initiated by $1-3$ days after surgery.

\section{Salvage surgery}

Summary The definition of salvage surgery is often debated. In the narrow sense, salvage surgery is aimed at curative resection of residual or recurrent tumor after definitive chemoradiotherapy. In Japan, the experience of salvage surgery began to be reported in the early 2000s. Although its indication and role have not yet been established, salvage surgery is recognized to pose a greater risk than general surgery for esophageal carcinoma. It is also known that the frequency of noncurative resection is high, resulting in a poor prognosis. In contrast, cases of curative resection are accompanied by an improved prognosis. Currently, no treatment other than salvage surgery (including endoscopic resection) is accepted as curative treatment for residual or recurrent tumor. Salvage surgery must be implemented only with the informed consent of the patients obtained after explaining the risks and long-term outcomes, and thus requires cautious consideration.

\section{Definition}

The 10th edition of the Japanese Classification of Esophageal Cancer defines salvage surgery as that for residual or recurrent cancer after definitive (chemo-) radiotherapy. The variety of surgeries include resection of the esophagus, removal of lymph nodes (dissection), and endoscopic resection (for salvage endoscopic treatment, see 
XI. Chemoradiotherapy). Additional resection of residual or recurrent tumor after endoscopic treatment may also be referred to as salvage surgery, but this is not included in these guidelines.

The standard radiation dose for definitive chemoradiotherapy is 50.4 Gy in Europe and North America, on the basis of the results of the INT0123 study. However, in Japan, many institutions use $60 \mathrm{~Gy}$ or more as the radiation dose for definitive chemoradiotherapy. The Japan Esophageal Society defines surgery following irradiation of $50 \mathrm{~Gy}$ or more as salvage surgery. Although salvage surgery is aimed at achieving curative resection, it ends up in non-curative resection at times because of its nature as a rescue treatment. Therefore, curativity (i.e., $\mathrm{R} 0$ resection or not) is not included in its definition.

\section{Indications}

The indication for salvage surgery is determined by both tumor factors and patient factors. Tumor factors include the feasibility of radical resection and the long-term prognosis, whereas patient factors include the patient's general ability to tolerate surgery, especially the functions of important organs such as the heart and lung.

The incidence of complications is higher in cases of salvage surgery than in patients treated by surgery alone or surgery combined with preoperative chemoradiotherapy (radiation dose less than $50 \mathrm{~Gy}$ ). The reported incidence of respiratory complications such as pneumonia is 9-62\%, while that of anastomotic leakage is 14-39\%. In particular, it is of great concern that the incidence of serious complications due to tissue ischemia, such as tracheal necrosis, perforation, and necrosis of the reconstructed gastric tube, is higher after salvage surgery than that after usual surgery. The reported in-hospital mortality after salvage surgery is $7-22 \%$, indicating that this type of surgery is associated with a higher surgical risk than usual surgery. The high incidence of complications and high in-hospital mortality should be taken into account when considering the indications for salvage surgery.

With regard to the postoperative survival rate, the reported 5-year survival rate is $25-35 \%$. Long-term survival can be expected in patients only when curative resection is achieved. However, the rate of non-curative resection is high in salvage surgery, reported to be in the range of $12-50 \%$. Because the prognosis of patients with non-curative resection is extremely poor, careful judgment is required when determining the indications for salvage surgery.

\section{Neoadjuvant therapy}

\section{Summary}

Neoadjuvant therapy (preoperative adjuvant therapy) has been compared with surgery alone or postoperative adjuvant therapy in cases of resectable Stage II or III thoracic esophageal carcinoma. The significance of neoadjuvant therapy for Stage I esophageal carcinoma has yet to be evaluated.

Neoadjuvant chemotherapy The randomized controlled trial (JCOG9907 study) that compared neoadjuvant chemotherapy and postoperative chemotherapy with cisplatin +5 -FU in patients with resectable Stage II or III thoracic esophageal carcinoma (2002 UICC classification) revealed a significant improvement in the overall survival time in the patients given neoadjuvant chemotherapy. Based on this finding, neoadjuvant chemotherapy + radical surgery for resectable Stage II or III thoracic esophageal carcinoma was positioned as a standard treatment in Japan.

Neoadjuvant chemoradiotherapy The results of a metaanalysis of randomized controlled trials performed in Europe and North America have indicated that neoadjuvant chemoradiotherapy combined with surgery has the potential to improve the long-term survival in patients undergoing surgical resection for esophageal carcinoma as compared to surgery alone. In Japan, this combination therapy is administered to patients with locally advanced carcinoma in some institutions, although, currently, there is no firm basis for its recommendation.

An expected advantage of neoadjuvant therapy over postoperative adjuvant therapy is that it is easier to complete the protocol treatment when applied as neoadjuvant therapy. Neoadjuvant therapy is expected to improve the resection rate and long-term follow-up results by reducing the size of the primary lesion and controlling lymph node metastasis and micrometastasis. It is possible that neoadjuvant therapy allows assessment of the patients' response to chemotherapy and radiotherapy to some extent by allowing histopathologic studies of the resected specimens. However, issues of concern include the following: drug resistance may be induced; local control may be delayed in ineffective cases, resulting in facilitation of metastatic spread of the disease; preoperative radiotherapy may make surgical manipulations more difficult and increase the postoperative risk.

The multicenter clinical study JCOG9907 carried out by the Esophageal Oncology Group of the Japan Clinical Oncology Group (JCOG) deserves special mention in the present revision of the guidelines, because its results provided the rationale for neoadjuvant chemotherapy to be established as the standard treatment for patients with no particular impediments to this treatment.

\section{Neoadjuvant chemotherapy}

A number of randomized controlled trials have been conducted in Europe and America addressing the possible beneficial effects of neoadjuvant chemotherapy on the survival rates of patients with esophageal carcinoma. According 
to the results of a meta-analysis of these randomized controlled trials, the effects of neoadjuvant chemotherapy on the survival of the patients varied according to the set end points. Therefore, the effects of neoadjuvant chemotherapy in resectable cases have not yet been established (T1-3N0, 1M0, UICC Classification, the 2002 edition).

The 2007 Guidelines for Diagnosis and Treatment of Carcinoma of the Esophagus recommended the implementation of neoadjuvant chemotherapy particularly in patients with positive lymph node metastasis, on the basis of the results of the JCOG9204 study (1992-1997: postoperative adjuvant chemotherapy with cisplatin +5 -FU vs. surgery alone). At the time of revising the guidelines, the timing of adjuvant chemotherapy with cisplatin +5 -FU was examined in the JCOG9907 study (1999-2006), and it was found that neoadjuvant chemotherapy yielded significantly improved overall survival in comparison to postoperative chemotherapy. Therefore, in Japan, neoadjuvant chemotherapy with cisplatin +5 -FU followed by radical surgery is now regarded as the standard treatment for resectable Stage II or III thoracic esophageal carcinoma.

\section{Neoadjuvant chemoradiotherapy}

Neoadjuvant chemoradiotherapy is a treatment strategy premised on planned surgery and is distinguished from rescue treatment for residual or recurrent carcinoma following chemoradiotherapy aimed at radical cure. In recent years, this type of treatment is recognized as a tri-modality therapy involving a combination of chemotherapy, radiotherapy, and surgery rather than as neoadjuvant chemoradiotherapy. In some cases, a radiation dose of $50.4 \mathrm{~Gy}$, which is the standard dose for definitive chemoradiotherapy, is used.

In Europe and North America, a number of randomized controlled trials that verified the usefulness of neoadjuvant chemoradiotherapy have been reported since the latter half of the 1980s, because of the limitations of local control by surgery. Although neoadjuvant chemoradiotherapy did not contribute to overall survival in most of these studies, the pCR rate was generally higher in patients given neoadjuvant chemoradiotherapy. In addition, in a study of esophageal adenocarcinoma reported by Walsh et al. and in the CALGB9781 study, the postoperative survival rate was significantly higher in patients given neoadjuvant chemoradiotherapy than in those undergoing surgery alone. In a randomized controlled trial conducted by Bosset et al. in patients with squamous cell carcinoma, neoadjuvant chemoradiotherapy significantly improved the recurrence-free survival period, although there was no prolongation of overall survival. Many other randomized controlled trials have demonstrated prolongation of survival in patients receiving neoadjuvant chemoradiotherapy, although there was no significant influence in the overall survival rate.

According to a meta-analysis that addressed surgery preceded by neoadjuvant chemoradiotherapy vs. surgery alone, when the 3-year survival rate was used as an end point, neoadjuvant chemoradiotherapy (20-45 Gy) in patients with resectable carcinoma was associated with a significant increase in operation-related mortality within 90 postoperative days. However, it resulted in a decrease in the local recurrence rate and significant increase of the 3-year survival rate.

In meta-analyses carried out so far in Europe and North America, the patient characteristics (histologic type, stage, etc.) and chemoradiotherapy protocols have not been consistent. The radicality of surgery has been suggested to greatly influence the outcome. No randomized controlled trials of neoadjuvant chemoradiotherapy have been carried out to date in Japan, and thus at present there is no satisfactory rationale for recommending this therapy as effective preoperative therapy.

\section{Postoperative adjuvant therapy}

\section{Summary}

Surgery combined with postoperative adjuvant therapy has been compared to surgery with or without preoperative adjuvant therapy in resectable cases or cases of Stage II or Stage III thoracic esophageal carcinoma judged as candidates for curative resection. However, to date, the value of postoperative adjuvant therapy for patients with Stage I esophageal carcinoma has not yet been studied.

Postoperative chemotherapy: A randomized controlled trial (JCOG9204 study) comparing surgery with and without postoperative chemotherapy (cisplatin +5 -FU, 2 courses) carried out in Japan demonstrated that postoperative chemotherapy resulted in a significant increase in the disease-free survival rate as compared to surgery alone, although there was no significant difference in the overall survival rate. Analysis of subgroups from the JCOG9204 study demonstrated that the recurrence-preventive effect of 2 courses of cisplatin +5 -FU therapy administered postoperatively was observed only in patients with positive lymph node metastasis. Therefore, in clinical practice, postoperative adjuvant chemotherapy has been considered only after carefully considering the pathological diagnosis after radical surgery. However, in view of the results of the aforementioned JCOG9907 study, implementation of neoadjuvant chemotherapy has been positioned as a standard treatment after taking into account the general condition of the patient. 
Postoperative radiotherapy: The results of a randomized controlled trial of pre- and postoperative radiotherapy vs. postoperative radiotherapy alone carried out by the Esophageal Oncology Group of the JCOG showed that the overall survival rate was significantly higher in patients given postoperative radiotherapy alone when the analysis was focused only on eligible patients who received treatment according to the protocol. Based on this finding, preventive postoperative irradiation was formerly in wide use in Japan. However, in overseas randomized controlled trials that compared surgery with and without postoperative irradiation (usual fractionation; 45-60 Gy), postoperative irradiation was associated with a decrease in the local recurrence in the irradiated area, but without a significant increase in the survival rate. Therefore, there is little evidence for recommending postoperative irradiation after curative resection as a standard treatment. At present, the significance of postoperative (chemo-) radiotherapy is unclear. (Chemo-) radiotherapy has been employed in clinical practice and also reported to be effective in cases of non-curative resection or postoperative local recurrence. Although there is insufficient evidence, some local therapy may be necessary for patients who have undergone non-curative resection and who have macroscopic residual tumor without distant metastasis. (Chemo-) radiotherapy seems to be a useful treatment option for such patients.

The rationale for implementing postoperative adjuvant therapy is its potential to control local residual tumor after surgical resection, lymph node metastasis outside the dissection area, and distant micrometastasis, and thereby to lead to improvement in long-term outcomes. The advantage of postoperative adjuvant therapy is that it is possible to implement treatment suited to the disease stage as determined at surgery. However, its disadvantages are that the absence of evaluable lesions makes it difficult to determine its efficacy and postoperative adjuvant therapy may be associated with a lower rate of completion of therapy than preoperative adjuvant therapy.

The 2007 Guidelines for Diagnosis and Treatment of Carcinoma of the Esophagus stated that radical resection followed by postoperative chemotherapy was the most commonly employed treatment strategy in Japan. However, preoperative chemotherapy (neoadjuvant chemotherapy) is now regarded as a standard treatment on the basis of the results of the aforementioned JCOG9907 study (1999-2006: neoadjuvant chemotherapy vs. postoperative chemotherapy).

\section{Postoperative chemotherapy}

In a randomized controlled trial (JCOG8806) in patients with squamous cell carcinoma carried out by the
Esophageal Oncology Group of the JCOG (comparison between surgery plus 2 courses of postoperative cisplatin + vindesine and surgery alone), addition of postoperative chemotherapy did not have any beneficial effect on survival and yielded no significant difference in the 5-year survival rate. Subsequently, another randomized controlled trial carried out in patients with esophageal squamous cell carcinoma using a different chemotherapy regimen (JCOG9204: comparison between surgery plus 2 courses of postoperative cisplatin +5 -FU and surgery alone) showed no clear intergroup difference in the overall survival rate. However, the disease-free survival time was significantly longer in patients given postoperative chemotherapy than in those treated by surgery alone. Thus, postoperative chemotherapy appeared to have a recurrence-preventive effect, particularly in patients with lymph node metastasis. By contrast, no such beneficial effect of postoperative adjuvant therapy was noted in patients without lymph node metastasis.

A randomized controlled trial carried out in France comparing surgery with and without 6-8 courses of postoperative cisplatin +5 -FU included patients undergoing palliative resection who accounted for about half of all the subjects. There was no significant difference in the median survival time between the two groups, and the researchers concluded that postoperative adjuvant chemotherapy with cisplatin and 5-FU exerted no beneficial effect. A meta-analysis of these randomized controlled trials also showed no beneficial effect of adjuvant chemotherapy on the survival rate.

The results of surgery with and even without adjuvant therapy obtained from all previous JCOG clinical studies were much better than the results of surgery plus postoperative adjuvant therapy reported by clinical studies conducted in Europe and North America. This may reflect the differences in the policy and accuracy of lymph node dissection between Japan and Western countries. This should be borne in mind when comparing the results of clinical studies carried out in Japan and those conducted in Europe and North America.

Thus, there is not enough evidence to show that adjuvant chemotherapy improves the overall survival rate of patients undergoing curative resection. However, a Japanese randomized controlled study (JCOG9204) demonstrated a significant increase in the disease-free survival rate after adjuvant chemotherapy, showing the recurrence-preventive effect of this therapy, particularly in patients with lymph node metastasis. Considering the fact that the accuracy of lymph node dissection is characteristically high in Japan and placing weight on the evidence obtained from this country, adjuvant chemotherapy (cisplatin +5 -FU, 2 courses) seems to have a role in the prevention of postoperative recurrence in patients with lymph node metastasis in whom curative resection has been achieved without any preoperative therapy. 


\section{Adjuvant radiotherapy}

Preoperative irradiation has long been considered a standard treatment in Japan. However, since there is no report definitively documenting that preoperative irradiation improves the survival rate, the Esophageal Oncology Group of the JCOG performed a randomized controlled trial from 1981 to 1984 to compare the outcomes of preoperative (30 Gy/15 fractions) plus postoperative ( $24 \mathrm{~Gy} / 12$ fractions) irradiation with postoperative irradiation alone (50 Gy/25 fractions). Since a number of cases were excluded from the analysis in this study because of non-curative resection or surgical complications, the results may be somewhat unreliable. However, when the analysis was confined to the eligible patients who received treatment as per protocol, the overall survival rate was significantly higher in the group administered postoperative irradiation alone. Based on this finding, prophylactic postoperative irradiation came to be used commonly in Japan.

By contrast, 4 overseas randomized controlled trials comparing surgery with and without postoperative irradiation (usual fractionation, 45-60 Gy) demonstrated no significant increase in the survival rate, although the rate of local recurrence in the irradiated areas decreased in the group given postoperative irradiation. A meta-analysis based on these controlled studies also showed no increase in the survival rate in the group treated by postoperative irradiation. Therefore, there is little evidence for recommending postoperative radiotherapy following curative resection as a standard treatment. However, a subset analysis in a large-scale randomized controlled trial performed in China showed that postoperative radiotherapy led to a significant increase in the survival rate in Stage III patients alone. Therefore, postoperative radiotherapy may be of clinical value for selected patients.

\section{Chemotherapy}

Summary

Chemotherapy in the treatment of esophageal carcinoma is usually combined with surgery or radiotherapy in the form of preoperative or postoperative adjuvant chemotherapy or chemoradiotherapy. The use of chemotherapy not combined with other modalities is limited to patients with distant metastasis (M1b) or postoperative distant recurrence. Currently, 5-FU + cisplatin is the most commonly used combination regimen for chemotherapy. However, since there is no definitive evidence of prolongation of the survival time, this therapy is regarded as a palliative treatment.

\section{Proven effective monotherapy drugs}

Many chemotherapeutic drugs, such as 5-FU, cisplatin, mitomycin $\mathrm{C}$, bleomycin, vindesine, adriamycin, paclitaxel, docetaxel, vinorelbine, nedaplatin, irinotecan, and gemcitabine are known to be effective in the treatment of esophageal carcinoma (Table 3). However, while 15-44 \% of patients have been estimated to respond to monotherapy, cases of complete response (CR) are rare and no monotherapy has been shown to have a survivalprolonging effect. At present, the most commonly used drugs are 5-FU and cisplatin. Basic studies have demonstrated that these two drugs are effective when used as monotherapy, and exert a synergistic effect when combined and a sensitizing effect when combined with radiotherapy. In clinical, many reports were published about good results of these drags combination therapy. These are the reasons for the wide use of these two drugs. At present (as of February 2012), the use of paclitaxel is not yet covered by the national health insurance in Japan, although public knowledge-based application of this drug has been approved. The use of vinorelbine, irinotecan, and gemcitabine for the treatment of esophageal carcinoma is also not covered by the national health insurance in Japan.

\section{Efficacy in combination therapy}

Although various combination therapies using cisplatin have been employed since this drug was introduced clinically (Table 4), the currently most commonly used
Table 3 Efficacy of major monochemotherapeutic agents against carcinoma of the esophagus (drugs covered by the national health insurance in Japan)

a This drug is approved through public knowledge-based application (as of February 2012)

\begin{tabular}{llll}
\hline Drug & Dose and schedule & No. of cases & Response rate (\%) \\
\hline 5 -FU & $500 \mathrm{mg} / \mathrm{m}^{2} /$ day $\times 5$ days & 26 & 15 \\
Mitomycin C & $20 \mathrm{mg} / \mathrm{m}^{2}$ every 4-6 weeks & 24 & 42 \\
Cisplatin & $50 \mathrm{mg} / \mathrm{m}^{2}$ every 3 weeks & 24 & 25 \\
Vindesine & $3-4.5 \mathrm{mg} / \mathrm{m}^{2}$ every week & 23 & 18 \\
Docetaxel & $70 \mathrm{mg} / \mathrm{m}^{2}$ every 3 weeks & 48 & 21 \\
Nedaplatin & $100 \mathrm{mg} / \mathrm{m}^{2}$ every 4 weeks & 29 & 52 \\
Paclitaxel $^{\mathrm{a}}$ & $100 \mathrm{mg} / \mathrm{m}^{2}$ every week $\times 6$, 2-week withdrawal & 52 & 44 \\
\hline
\end{tabular}


Table 4 Efficacies of major combination therapies

\begin{tabular}{|c|c|c|c|}
\hline Drug & Histologic type & No. of cases & Response rate $(\%)$ \\
\hline $5-\mathrm{FU}+$ cisplatin & Squamous cell carcinoma & 39 & 36 \\
\hline Cisplatin + paclitaxel $^{\mathrm{a}}$ & Squamous cell carcinoma/adenocarcinoma & 32 & 44 \\
\hline Cisplatin + irinotecan $^{\mathrm{b}}$ & Squamous cell carcinoma/adenocarcinoma & 35 & 57 \\
\hline Cisplatin + gemcitabine $^{b}$ & Squamous cell carcinoma/adenocarcinoma & 32 & 45 \\
\hline $5-\mathrm{FU}+$ nedaplatin & Squamous cell carcinoma & 38 & 40 \\
\hline
\end{tabular}

a This regimen is approved through public knowledge-based application (as of February 2012)

b This regimen is not approved for insurance coverage (as of February 2012)

combination regimen is 5 -FU + cisplatin. In other countries, this combination therapy is usually administered as follows: continuous intravenous infusion of 5-FU at $1,000 \mathrm{mg} / \mathrm{m}^{2} /$ day for $4-5$ days, plus intravenous cisplatin at $100 \mathrm{mg} / \mathrm{m}^{2}$ on day 1 . In contrast, a phase II clinical trial of 5-day continuous intravenous infusion of 5-FU at $700 \mathrm{mg} / \mathrm{m}^{2} /$ day plus intravenous cisplatin at $70 \mathrm{mg} / \mathrm{m}^{2}$ on day 1 carried out in Japan showed a response rate of $36 \%$. A comparison between this combination therapy and best supportive care carried out overseas demonstrated no definite prolongation of survival in the former group. However, this study included many patients in whom this therapy was used mainly as adjuvant chemotherapy. However, it excluded those in whom the metastatic focus in the liver accounted for more than $30 \%$ of the hepatic parenchyma and those who had peritoneal dissemination. Therefore, the effect of this combination therapy on survival remains unclear. Although in recent years, regimens containing paclitaxel, irinotecan, or gemcitabine have been tried overseas, and regimens using nedaplatin or docetaxel have been tried in Japan, no large-scale phase III trials of these regimens have been carried out. Thus, the merits of these regimens over the standard combination of 5-FU + cisplatin have yet to be demonstrated. Currently in Japan, the combination of 5-FU + cisplatin is commonly used as the first-line treatment, followed by docetaxel as a second-line treatment. In any event, the effect of the use of chemotherapy alone, regardless of whether it is combination therapy or monotherapy, is limited, and chemotherapy not combined with other treatment modalities is applied only to patients with unresectable metastatic lesions.

Cisplatin, a chemotherapeutic drug that is in wide use, is classified as a highly pro-emetic drug. Guidelines for appropriate use of antiemetic drugs recommend the triple-drug combination of a $5-\mathrm{HT}_{3}$ receptor antagonist, corticosteroid, and aprepitant to prevent emesis while using cisplatin. For other drugs, it is necessary to check the risk of emesis against guidelines for appropriate use of antiemetic drugs and to take appropriate prophylactic measures.

\section{Radiotherapy}

Summary

As compared to radiation monotherapy, concurrent chemoradiotherapy significantly increases the survival rate, although radiotherapy administered sequentially after induction chemotherapy does not. Concurrent chemoradiotherapy is indicated for patients with T1-4N0-3M0 carcinoma (UICC-TNM classification, 2009 edition) in good general condition and for those with locally advanced carcinoma up to metastasis to the supraclavicular lymph nodes (M1). However, the risk of serious complications such as fistula formation is high in cases of unresectable locally advanced carcinoma (T4).

Because prolongation of the duration of irradiation decreases the local control rate of radiation monotherapy, it is important to complete irradiation using a definitive dose (66-68.4 Gy) within 7 weeks. In definitive concurrent chemoradiotherapy, the use of at least $50 \mathrm{~Gy} / 25$ fractions $/ 5$ weeks with a conventional fractionation protocol is necessary. The standard radiation dose for concurrent chemoradiotherapy in the USA is $50.4 \mathrm{~Gy} / 28$ fractions. In contrast, in Japan, the standard radiation dose is $60 \mathrm{~Gy} / 30$ fractions/6-8 weeks for concurrent chemoradiotherapy, and its safety has already been demonstrated.

A randomized controlled trial carried out in Japan revealed that concomitant use of external beam radiation and intracavitary radiation is effective for patients with T1-2 esophageal carcinoma, a relatively early stage of the disease. However, recently chemoradiotherapy is used commonly, and the available evidence is not sufficient to recommend the addition of intracavitary radiation to chemoradiotherapy.

Previously, radiotherapy was primarily used for patients who were not suitable candidates for surgery or endoscopic mucosal resection (EMR). However, in recent years, radiotherapy (in particular, chemoradiotherapy) has been widely used for both superficial carcinoma and locally advanced carcinoma, as definitive treatment. 
The standard radiotherapy used for esophageal carcinoma is in accordance with the Radiotherapy Planning Guidelines 2008 (ed. by Japanese College of Radiology, The Japanese Society for Therapeutic Radiology and Oncology, and Japan Radiological Society). The key points are described below.

Definitive radiotherapy

\section{Indications}

A definitive radiotherapy protocol is used when control of all gross lesions leading to cure is expected. Definitive irradiation is the most suitable for cases with T1-4N0-3M0 carcinoma (UICC-TNM classification, 2009 edition) and cases with locally advanced disease up to metastasis to the supraclavicular nodes (M1). In patients with a favorable general condition allowing combined use of chemotherapy, the standard treatment is chemoradiotherapy rather than radiation monotherapy.

Definitive chemoradiotherapy has been used for the treatment of postoperative recurrence in the regional lymph nodes in patients without distant metastasis or postoperative residual tumor, as well as for definitive irradiation in fresh cases. It has been shown to provide favorable therapeutic results.

\section{Target volume}

Gross tumor volume (GTV) GTV includes the esophageal primary foci (GTV primary) and metastatic lymph nodes (GTV nodal) as determined by endoscopy and CT. In cases of esophageal carcinoma, it is difficult to determine the presence of lymph node metastasis on the basis of the sizes of the lymph nodes. However, it has been reported to be relatively safe to treat lymph nodes measuring $5 \mathrm{~mm}$ or more in the minor axis as determined by CT or MRI, regarding them as metastatic foci, to decrease the percentage of false-negative cases.

${ }^{18} \mathrm{~F}$-fluorodeoxyglucose (FDG)-PET is useful for staging because it allows the detection of hidden distant metastases. However, the sensitivity and specificity of this technique to detect metastatic lymph nodes are not always high in cases of esophageal carcinoma; therefore, it may not be reasonable to attempt to identify metastatic lymph nodes on the basis of PET findings alone for treatment planning. On the positive side, it has been reported that PET/CT can confirm the extent of the primary focus (GTV primary) better than those based on CT alone, if the threshold of FDG activity is set properly.

Clinical target volume 1 (CTV1) CTV1 is defined as the entire circumference of the esophagus including the GTV primary on endoscopy or CT, as well as possible microscopic lesions within 3-4 cm cephalocaudally and regional lymph nodes. A study of resected specimens of esophageal squamous cell carcinoma in 34 patients showed that the mean extent of microscopic invasion from the GTV primary was $10.5 \pm 13.5 \mathrm{~mm}$. It has been reported that a CTV margin of $3 \mathrm{~cm}$ would cover the aforementioned extent of microscopic progression in $94 \%$ of patients.

However, because EP and LPM lesions in cases of T1a carcinoma rarely metastasize to the lymph nodes, irradiation of regional lymph nodes is not required. By contrast, lymph node metastasis is present in 10-50\% of cases of MM or SM superficial carcinomas. Therefore, prophylactic irradiation of the regional lymph nodes is required for these cases, as for cases of advanced esophageal carcinoma. Table 5 lists the standard CTV1 in relation to the site of the primary lesion. Currently, there is not enough evidence and there is no evidence-based consensus on which lymph node regions the CTV should extend to. The irradiation dose to these areas should be 40-46 Gy/20-23 fractions. In regard to chemoradiotherapy for superficial esophageal carcinoma (cT1N0M0), it has been reported that favorable therapeutic results can be obtained by localized irradiation, allowing a 3-cm margin inferiorly and superiorly, and a $1-$ to $2-\mathrm{cm}$ margin anteriorly, posteriorly, and on both sides of the primary focus (GTV primary). Thus, extensive irradiation of regional lymph nodes may not be necessary for superficial esophageal carcinoma.

Clinical target volume 2 (CTV2) CTV2 after irradiation of 40-46 Gy to CTV1 should cover the whole circumference of the esophagus including the GTV primary with addition of a margin of about $2 \mathrm{~cm}$ in the cephalocaudal direction and $0-0.5 \mathrm{~cm}$ in the lateral direction, and the area of metastatic lymph nodes (GTV nodal) with an additional margin of about $0-0.5 \mathrm{~cm}$ in the lateral direction.

Planning target volume 1 (PTV1) The planning target volume at the beginning of radiotherapy (PTV1) should include CTV1 with an adequate margin $(0.5-1.0 \mathrm{~cm}$ in the lateral direction and $1-2 \mathrm{~cm}$ in the cephalocaudal direction), allowing for respiratory movements and errors in reproducing the patient's fixation. Because respiratory movements are particularly large in the case of lower thoracic esophageal carcinoma, a margin of $0.8 \mathrm{~cm}$ in the lateral direction and $1.8 \mathrm{~cm}$ in the cephalocaudal direction has been reported to be necessary.

Planning target volume 2 (PTV2) The planning target volume for the reduced exposure field at 40-46 Gy (PTV2) should include CTV2 with an adequate margin $(0.5-1.0 \mathrm{~cm}$ in the lateral direction and $1-2 \mathrm{~cm}$ in the cephalocaudal direction). 
Table 5 Standard lymph node regions in relation to the site of the primary focus (CTV1)

\begin{tabular}{|c|c|}
\hline Cervical esophagus (Ce) & $\begin{array}{l}\text { From the midsdle deep cervical lymph nodes [102-mid] to the lymph nodes at the tracheal } \\
\text { bifurcation [107] }\end{array}$ \\
\hline Upper thoracic esophagus (Ut) & $\begin{array}{l}\text { From the supraclavicular lymph nodes [104] to the middle thoracic paraesophageal lymph } \\
\text { nodes [108] }\end{array}$ \\
\hline Middle thoracic esophagus (Mt) & $\begin{array}{l}\text { a. From the supraclavicular lymph nodes [104] to the lower thoracic paraesophageal [110] } \\
\text { or perigastric lymph nodes } \\
\text { b. From the lymph nodes along the recurrent laryngeal nerve [106-rec] and upper thoracic } \\
\text { paraesophageal lymph nodes [105] to the lower thoracic paraesophageal [110] or per- } \\
\text { igastric lymph nodes }\end{array}$ \\
\hline Lower thoracic esophagus (Lt) & $\begin{array}{l}\text { From the lymph nodes along the recurrent laryngeal nerve [106-rec] and upper thoracic } \\
\text { paraesophageal lymph nodes [105] to the perigastric lymph nodes }\end{array}$ \\
\hline Patients of advanced age or with complications & Only lymph node regions around the primary focus \\
\hline
\end{tabular}

Locations of regional lymph nodes of the esophagus on CT images are shown in the 10th edition of the Japanese Classification of Esophageal Cancer

Perigastric lymph nodes: Cardiac lymph nodes [1,2], lymph nodes along the lesser curvature [3], and lymph nodes along the left gastric artery [7]

There is no consistent consensus on CTV1 in cases of primary carcinoma originating in the middle thoracic esophagus (Mt)

\section{Radiotherapy planning and the irradiation method}

Three-dimensional treatment planning based on CT images is recommended. This method allows an understanding of the 3-dimensional positional relationship between the target volume and the organs at risk, and is useful for implementing high-accuracy radiotherapy to minimize exposure of the organs at risk. If a lesion cannot be visualized by $\mathrm{CT}$, as in the case of superficial carcinoma, clipping to the upper and lower parts of the lesion under endoscopic guidance is required prior to $\mathrm{CT}$ imaging. Organs at risk that require particular attention in treatment planning for esophageal carcinoma include the lung, heart, and spinal cord.

The use of 6-10 MV X-rays is recommended for external irradiation. An appropriate point in the PTV is chosen as the dose assessment point. Irradiation should be applied while restricting the cumulative maximum dose to the spinal cord to 44-46 Gy or less by using the fixed multiple field technique or by changing the radiation field midcourse. Intensity-modulated radiotherapy (IMRT) may be used for the treatment of cervical esophageal carcinoma at facilities that have radiation oncologists and medical physicists who are familiar with treatment planning to secure adequate quality control of radiotherapy.

\section{Dose fractionation}

In general, the conventional fractionation method is used. The standard radiation dose for chemoradiotherapy used overseas is about $50 \mathrm{~Gy} / 25-28$ fractions/5-6 weeks. In contrast, in Japan, the standard radiation dose is about $60 \mathrm{~Gy} / 30$ fractions/6-8 weeks for chemoradiotherapy, and 60-70 Gy/30-35 fractions/6-7 weeks for radiation monotherapy. Chemoradiotherapy is described in detail in chapter XI.

The overall treatment time is an important factor in radiotherapy for esophageal squamous cell carcinoma. The local control rate is known to decrease with increase in the overall treatment time of radiotherapy; therefore, in cases of radiation monotherapy, it is important to avoid prolongation of the overall treatment time as much as possible.

\section{Intracavitary radiation}

In Japan, superficial esophageal carcinoma is considered to be a suitable indication for intracavitary irradiation, because this technique of irradiation can deliver sufficient radiation dose to the superficial lesions. A retrospective analysis from a single institution indicated that a radiation boost by intracavitary irradiation for a superficial lesion of the esophagus yielded favorable therapeutic results. However, the report by Nemoto et al., who reviewed multicenter studies carried out in Japan, indicated that there was no difference in the survival rate between external radiation monotherapy and external radiotherapy combined with intracavitary irradiation in patients with superficial carcinoma of the esophagus. While randomized controlled trials focusing on superficial esophageal carcinoma have never been done, randomized controlled trials of intracavitary irradiation for esophageal carcinoma, including advanced cases in Japan, reported that intracavitary irradiation was effective for esophageal carcinomas measuring $5 \mathrm{~cm}$ or less in the major axis or those with a depth of invasion corresponding to $\mathrm{T} 1$ or $\mathrm{T} 2$. However, more recently, chemoradiotherapy has come to be used commonly, and the efficacy and safety of an additional intracavitary radiation boost to chemoradiotherapy are not necessarily clear. 
For intracavitary irradiation, a balloon applicator measuring $15-20 \mathrm{~mm}$ in diameter should be used to avoid uneven distribution of the radiation source. The point of dose assessment should be $5 \mathrm{~mm}$ lateral to the applicator surface ( $5 \mathrm{~mm}$ submucosal), and the dose on the mucosal surface should also be reported. Although there is no definite consensus about the optimal dose and fractionation of intracavitary irradiation because they are closely related to the combined external irradiation dose, the general practice is external irradiation at 50-60 Gy followed by intracavitary irradiation at 8-12 Gy/2-4 fractions (3-4 Gy per session). Because an increase in the fractional dose of intracavitary radiation is associated with an increased risk of late complications, such as esophageal ulcers and perforation, 1-2 sessions per week at a dose of $4 \mathrm{~Gy}$ or less per session for high-dose-rate irradiation, or at a dose of 6 Gy or less per session for low-dose-rate irradiation is recommended.

\section{Complications}

Major early adverse events include radiation dermatitis, radiation esophagitis, and radiation pneumonia. Radiation esophagitis is almost inevitable; however, the possibility of development of esophagomycosis or reflux esophagitis should also be borne in mind. Radiation pneumonia, which is sometimes a serious issue, requires differentiation from infectious pneumonia and carcinomatous lymphangitis.

As late adverse events, esophageal perforation and bleeding occur in some patients treated by radiotherapy. The incidence rate of late adverse events is relatively increased in cases of T4 disease. In cases where high-dose intracavitary irradiation is employed, special caution concerning the occurrence of esophageal ulcers and perforation is necessary. The incidence of these conditions has been reported to be increased in patients given intracavitary irradiation after chemoradiotherapy. Esophageal stenosis may occur in patients with circumferential disease or those subjected to repeated EMR. Because radiation pneumonia may be fatal in patients of advanced age, it is necessary to reduce the exposure dose to the lung in treatment planning. The possibility of thoracic vertebral compression fracture within the radiation field requires particular attention and should be differentiated from bone metastasis.

Pericardial effusion and constrictive pericarditis associated with radiation epicarditis and pleural effusion caused by radiation pleuritis have been reported to occur at high frequencies after chemoradiotherapy. Radiation myelitis is a serious and rare late complication. There are case reports of radiation myelitis developing even in cases with an exposure dose to the spinal cord of only $44 \mathrm{~Gy}$, suggesting the need for particular vigilance regarding this complication. In addition, irradiation of the cervical area may cause hypothyroidism a few years post-irradiation. Because hypothyroidism may serve as a risk factor for radiation epicarditis and radiation pleuritis, regular monitoring of the thyroid functions is necessary in long-surviving patients.

Radiotherapy for symptomatic relief

This type of radiotherapy is aimed at improving the subjective symptoms and QOL and not at obtaining an anticancer effect. Radiotherapy may be used for the primary focus to improve dysphagia in patients with esophageal carcinoma, or for the treatment of distant metastases such as bone metastasis and brain metastasis. Although intracavitary irradiation monotherapy has been suggested to be useful for improving dysphagia, this radiotherapeutic technique is seldom used for the management of dysphagia associated with esophageal carcinoma in Japan.

For palliative irradiation, it is important to set the minimum necessary radiation field and total dose to achieve the treatment objective. The treatment should be completed within as short a period of time as possible, considering the general condition of the patient.

\section{Chemoradiotherapy}

\section{Summary}

Randomized controlled studies have demonstrated that chemoradiotherapy yields a significantly higher survival rate than radiation monotherapy in patients with esophageal carcinoma; therefore, this therapeutic modality is regarded as the standard therapy for patients with esophageal carcinoma when non-surgical treatment is the choice. Patients who can be the target of definitive chemoradiotherapy include T1-3N0-3M0 cases (UICC-TNM classification, 2009 edition), unresectable T4N0-3M0 cases, and cases with locally advanced disease up to metastasis to the supraclavicular nodes (M1). Some reports showed no significant difference in the overall survival and recurrence-free survival between patients with resectable lesions treated by chemoradiotherapy or by surgery alone. However, in Japan, neoadjuvant chemotherapy followed by surgery is considered to be superior to chemoradiotherapy in patients with Stage IB-III disease (UICC-TMN classification, 2009 edition), while the equivalence of chemoradiotherapy and surgery is considered in patients with Stage IA disease (T1N0M0, UICC-TNM classification, 2009 edition). Although the drug doses, radiation doses, and treatment schedules vary among different clinical studies, the most common protocol employed is combined chemotherapy with 5-FU plus cisplatin and concurrent radiotherapy at a total dose of 50-60 Gy. It is necessary to recognize that any of the reported treatment results can be reproducible only 
when the chemotherapy and radiotherapy defined in the study are adequately applied.

\section{Radiation dose in definitive chemoradiotherapy}

In a randomized controlled trial of radiation monotherapy (64 Gy) and concurrent chemoradiotherapy (5-FU + cisplatin + radiation $50 \mathrm{~Gy}$ ) for T1-4N0-1M0 esophageal carcinoma (corresponding to UICC-TNM classification, 2002 edition) carried out by the US Radiation Therapy Oncology Group (RTOG), the 5-year survival rate was $0 \%$ for the former and $26 \%$ for the latter; the latter treatment yielded significantly better results $(p<0.0001)$. Thus, chemoradiotherapy is strongly recommended in non-surgical treatment. In regard of the timing of chemotherapy and radiotherapy, a meta-analysis showed that concurrent chemoradiotherapy is associated with a significantly lower mortality rate $(p<0.0001)$ than sequential chemoradiotherapy. In addition, a randomized controlled study (RTOG9405/INT0123) carried out succeedingly to RTOG 85-01 that compared chemoradiotherapy using standard-dose (50.4 Gy) and high-dose $(64.8 \mathrm{~Gy})$ radiation in patients with T1-4N0-1M0 esophageal carcinoma (corresponding to UICC-TNM classification, 2002 edition) revealed no superiority of highdose radiation over standard-dose radiation in terms of the median survival time, the 2-year survival rate, and the local control rate, and concluded that the standard radiation dose for chemoradiotherapy using a combination of 5-FU plus cisplatin should be $50.4 \mathrm{~Gy}$ (1.8 Gy $\times 28$ times). By contrast, a radiation dose of $60 \mathrm{~Gy}$ has been used predominantly in studies carried out in Japan. Although the standard radiation dose has not yet been established in Japan, change to $1.8 \mathrm{~Gy} /$ fraction $\times 28$ times (total dose of $50.4 \mathrm{~Gy}$ ) is now under review at some facilities. For information on the method of irradiation and dose fractionation, see "Radiotherapy".

\section{Chemotherapy used in definitive chemoradiotherapy}

The standard chemotherapy regimen is 5-FU + cisplatin. In the aforementioned RTOG9405/INT0123 study, a course of 4 days' continuous intravenous infusion of 5 -FU at $1,000 \mathrm{mg} / \mathrm{m}^{2} /$ day plus intravenous cisplatin at $75 \mathrm{mg} / \mathrm{m}^{2}$ on day 1 was repeated every 4 weeks up to a total of 4 courses (concurrent radiation was used in the initial 2 courses). In Japan, although use of the 5-FU + cisplatin regimen is variable, a phase II clinical study (JCOG9708) of chemoradiotherapy $(5-\mathrm{FU}+$ cisplatin + irradiation of $60 \mathrm{~Gy})$ for cases of Stage I esophageal carcinoma (T1NOM0, UICCTNM classification, 1997 edition [*corresponding to Stage IA: T1N0M0 in the 2009 edition]) conducted by the JCOG used 2 courses of 4 days' continuous intravenous drip infusion of 5 -FU at $700 \mathrm{mg} / \mathrm{m}^{2} /$ day plus intravenous drip infusion of cisplatin at $70 \mathrm{mg} / \mathrm{m}^{2}$ on day 1 repeated every 4 weeks. In the JCOG9708 study, the complete response rate was $87.5 \%$, the 4-year survival rate was $80.5 \%$, and the 4-year progression-free survival rate was $68 \%$, suggesting results equivalent to those of surgery. Currently, a phase III clinical study (JCG0502) comparing chemoradiotherapy with surgery is underway. In another phase II JCOG study (JCOG9906) of chemoradiotherapy (5-FU + cisplatin + irradiation of $60 \mathrm{~Gy}$ ) performed in cases of resectable Stage II-III esophageal carcinoma, a course of 5 days' continuous intravenous infusion of $5-\mathrm{FU}$ at $400 \mathrm{mg} / \mathrm{m}^{2} /$ day for 2 weeks plus intravenous cisplatin at $40 \mathrm{mg} / \mathrm{m}^{2}$ on days 1 and 8 was repeated every 5 weeks for a total of 4 courses (the initial 2 courses were combined with concurrent irradiation). However, the use of chemotherapy according to the RTOG regimen is now under consideration in Japan. In any case, 2 courses of concurrent chemoradiotherapy are commonly administered. Although the use of additional chemotherapy after chemoradiotherapy is variable; 2 courses of additional chemotherapy are often administered for Stage II-III lesions. Table 6 shows the main schedules used in definitive chemoradiotherapy.

\section{Adverse events associated with definitive chemoradiotherapy}

Adverse events associated with chemoradiotherapy may be attributable to chemotherapy, radiotherapy, or both, and it is difficult to strictly distinguish among these causes. Major early adverse events include nausea, vomiting, myelosuppression, esophagitis, stomatitis, diarrhea, constipation, and radiation pneumonitis. In particular, radiation pneumonitis may be fatal, and it is desirable to identify factors that may predict the development of this condition. In this regard, it has been suggested that dose-volume histogram (DVH) parameters of irradiation may be useful. On the other hand, late adverse events include radiation pericarditis, radiation pleuritis, pleural effusion, and pericardial effusion. Hypothyroidism may occur in patients who have received radiation in the cervical area, which may also be accompanied by pleural effusion or pericardial effusion, necessitating caution. Although rare, the occurrence of thoracic vertebral compression fracture or radiation myelitis has also been reported (see "Radiotherapy"). In regard of the late toxic effects, it is considered that the radiation dose to organs at risk such as the lung and heart should be carefully considered. Use of a 3-dimensional radiation planning technique based on CT images aimed at reducing the toxic effects is now common.

Among other possible adverse events during chemoradiotherapy for esophageal carcinoma, special attention should be paid to the syndrome of inappropriate secretion of antidiuretic hormone (SIADH) attributable to cisplatin and leukoencephalopathy attributable to 5-FU. Early 
Table 6 Main schedules used in definitive chemoradiotherapy

\begin{tabular}{|c|c|c|c|c|c|c|}
\hline \multirow[t]{2}{*}{ Author } & \multirow[t]{2}{*}{ Target stage } & \multicolumn{2}{|l|}{ Chemotherapy drugs } & \multicolumn{3}{|l|}{ Radiation dose } \\
\hline & & $5-\mathrm{FU}$ & Cisplatin & $\begin{array}{l}\text { Period } \times \text { No. } \\
\text { of courses }\end{array}$ & $\begin{array}{l}\text { Single dose } \\
\times \text { No. of sessions }\end{array}$ & Split \\
\hline RTOG & $\mathrm{T} 1-4 \mathrm{~N} 0,1 \mathrm{M} 0$ & $1000 \mathrm{mg} / \mathrm{m}^{2} /$ day $\times 4$ days & $75 \mathrm{mg} / \mathrm{m}^{2}$ & every 4 weeks $\times 4$ & $1.8 \mathrm{~Gy} \times 28$ & None \\
\hline JCOG9708 & T1N0M0 & $700 \mathrm{mg} / \mathrm{m}^{2} /$ day $\times 4$ days & $70 \mathrm{mg} / \mathrm{m}^{2}$ & every 4 weeks $\times 2$ & $2.0 \mathrm{~Gy} \times 30$ & 1 week \\
\hline JCOG9906 & $\mathrm{T} 1 \mathrm{~N} 1 \mathrm{M} 0$ or $\mathrm{T} 2-3 \mathrm{~N} 0-1 \mathrm{M} 0$ & $400 \mathrm{mg} / \mathrm{m}^{2} /$ day $\times 10$ days & $40 \mathrm{mg} / \mathrm{m}^{2} \times 2$ & every 4 weeks $\times 2$ & $2.0 \mathrm{~Gy} \times 30$ & 2 week \\
\hline Ohtsu & T4/M1/LYM & $400 \mathrm{mg} / \mathrm{m}^{2} /$ day $\times 10$ days & $40 \mathrm{mg} / \mathrm{m}^{2} \times 2$ & every 5 weeks $\times 2$ & $2.0 \mathrm{~Gy} \times 30$ & 2 weeks \\
\hline Nishimura & $\mathrm{T} 4 \mathrm{M} 0$ & $300 \mathrm{mg} / \mathrm{m}^{2} /$ day $\times 14$ days & $10 \mathrm{mg} / \mathrm{m}^{2}$ & every 4 week $\times 2$ & $2.0 \mathrm{~Gy} \times 30$ & 1 week \\
\hline JCOG0303 & T4/M1LYM & $700 \mathrm{mg} / \mathrm{m}^{2} /$ day $\times 4$ days & $70 \mathrm{mg} / \mathrm{m}^{2}$ & every 4 weeks $\times 2$ & $2.0 \mathrm{~Gy} \times 30$ & 1 week \\
\hline KROSG0101 & Stage II-IVA & $700 \mathrm{mg} / \mathrm{m}^{2} /$ day $\times 5$ days & $70 \mathrm{mg} / \mathrm{m}^{2}$ & every 4 weeks $\times 2$ & $2.0 \mathrm{~Gy} \times 30$ & 1 week \\
\hline Nakajima & Stage II/III & $1,000 \mathrm{mg} / \mathrm{m}^{2} /$ day $\times 4$ days & $75 \mathrm{mg} / \mathrm{m}^{2}$ & every 4 weeks $\times 4$ & $1.8 \mathrm{~Gy} \times 28$ & None \\
\hline
\end{tabular}

Schedules without radiation split are being adopted in several ongoing clinical trials in Japan

detection and prompt medication, particularly prompt discontinuation of medication if the patient is on some drug therapy, are necessary.

\section{Follow-up after therapy}

Contrast-enhanced CT and endoscopic examination are generally used for follow-up observation after definitive chemoradiotherapy. Although there is no definitive evidence for the appropriate timing of the response evaluation and follow-up observation, patients are usually examined 3-4 weeks after completion of chemoradiotherapy at the end of each course of additional chemotherapy, and subsequently every 3 months during the first year, and every 4-6 months thereafter. Residual carcinoma or recurrence after chemoradiotherapy is found most frequently in the primary tumor in the esophagus and in the lymph nodes, usually within 1-2 years after the start of therapy. Therefore, if salvage therapy is considered, evaluation of the primary site is important. The initial endoscopic evaluation of the primary site within 75-90 days after the start of chemoradiotherapy, followed by a second evaluation within 1 month of the initial evaluation, is considered to be most effective in determining the presence/absence of exacerbation and judging whether a CR has been achieved. Because patients with esophageal carcinoma are known to show a relatively high likelihood of developing multiple carcinomas, with carcinomas developing de novo in other parts of the esophagus or in other organs (head and neck region, stomach, large intestine), careful follow-up observation and appropriate diagnostic measures are required.

\section{Salvage therapy for local remnant or recurrent lesions after definitive chemoradiotherapy}

Salvage therapy using endoscopy or surgery has recently been tried for the treatment of local remnant or recurrent lesions after definitive chemoradiotherapy (see "Salvage surgery"). As for salvage endoscopic treatment, endoscopic mucosal resection (EMR), endoscopic submucosal dissection (ESD), and photodynamic therapy (PDT) have been tried, and favorable long-term results with acceptable safety have been reported. However, the indications for these treatments and selection of the appropriate treatment method have not yet been adequately evaluated. Salvage surgery (see "Salvage surgery") provides cure in some cases; however, the incidence of operation-related adverse events and operation-related mortality is high, and the optimal surgical technique and extent of lymph node dissection have not yet been established. Therefore, salvage surgery is not employed in general practice. It has been reported that long-term survival may be achieved after salvage surgery when the depth of invasion of the residual or recurrent lesion is shallow, or when there is no residual or recurrent lymph node metastasis. To facilitate early detection of remnant or recurrence of the primary focus after definitive chemoradiotherapy, strict follow-up by endoscopy is necessary, bearing in mind the observations that the greater the $\mathrm{T}$ factor of the pretreatment staging, the more likely recurrence is, and that recurrence often occurs in the form of submucosal tumor-like elevation.

\section{Diagnosis and treatment of Barrett's esophagus and Barrett's carcinoma}

Summary

Esophagus showing Barrett's mucosa is called Barrett's esophagus. Barrett's mucosa refers to the columnar epithelial metaplasia that extends from the stomach to the esophagus in a continuous fashion and can be confirmed by endoscopy. Histological confirmation of specific columnar epithelial metaplasia is not required. Histologically, 
Barrett's mucosa exhibits one of the following features: (1) proper esophageal glands or ducts beneath the overlying columnar epithelium; (2) squamous epithelial islets in the columnar epithelium; (3) double structure of the lamina muscularis mucosae. Barrett's carcinoma is defined as adenocarcinoma occurring in Barrett's mucosa. The definitions of early, superficial, and advanced carcinomas are the same as those of esophageal carcinoma, regarding the deep-seated lamina muscularis mucosae as the original lamina muscularis mucosae. Treatment of Barrett's carcinoma is planned in accordance with the treatment of squamous cell carcinoma of the esophagus at the same location in the esophagus. Endoscopic resection is indicated for lesions confined to the lamina propria mucosae (EP, SMM, and LPM). Relative indications are currently under consideration.

\section{Diagnosis and treatment of double carcinoma (head and neck, stomach)}

\section{Summary}

Patients with esophageal carcinoma frequently develop carcinoma of other organs, particularly of the upper aerodigestive tract, including head and neck carcinoma, gastric carcinoma, and lung carcinoma. Preoperative examination and postoperative follow-up must be carried out bearing in mind the possible presence of double/multiple lesions. Therapeutic strategies and problems involved in treatment vary widely according to the type, stage, and time of onset of the other lesions. It is important to select the surgical technique and the treatment method in a well-balanced manner, taking into consideration the general condition of the patient, and the prognosis of the esophageal lesions and second primary lesions.

Double carcinoma is defined as the co-existence of two primary carcinomas in different organs. Patients with esophageal lesion are reported to show a higher incidence of double carcinoma as compared to the incidence of malignancy in the general population. This higher incidence of double carcinoma in esophageal carcinoma patients may be explained by the sharing of risk factors of carcinomas of the upper aerodigestive tract. We would also like to emphasize the concept of field cancerization here.

The incidences and types of double carcinoma vary according to the year of survey, the duration of observation, and the specialization level of the facilities. According to the national registry of the Japan Esophageal Society, about $20 \%$ of patients with esophageal lesion have a second primary lesion, being synchronous in $8 \%$ and metachronous in $12.2 \%$ of the cases. The most frequent type of double carcinoma was gastric lesions, followed by head and neck lesions (pharyngeal carcinoma), colorectal lesions and lung lesions, in descending order of frequency.

In addition, according to the 2007 statistics of the Japanese Association for Thoracic Surgery, the incidence of double carcinoma, including second primary carcinomas preceding esophageal carcinoma but excluding the ones following esophageal carcinoma, was $12.9 \%$, with the proportion of synchronous double lesions at $7.45 \%$. The type of double lesions was most frequently gastric lesions, followed by head and neck lesions.

From the viewpoint of diagnosis and treatment of esophageal lesions, the presence/absence of a second primary head and neck lesions is an important issue. A number of studies on double carcinoma in the esophagus and the head and neck region have so far been reported. Some reports claim that head and neck carcinomas may be the most frequently occurring second carcinoma in association with esophageal carcinoma.

Among patients with esophageal lesions, most studies report a frequency of head and neck lesions of about $10 \%$, with pharyngeal lesions the most frequent type of head and neck lesions encountered in these cases. Some characteristic features that may help in predicting the presence of head and neck lesions in patients with esophageal lesions include the presence of multiple esophageal lesions and the existence of multiple zones in the esophagus showing negative iodine staining.

In recent years, advances in endoscopic techniques, including magnifying endoscopy and image-enhanced endoscopy and increased attention to the head and neck region in patients with esophageal carcinoma, have increased the detection rate of head and neck carcinoma in the early stage by endoscopic examination of the upper gastrointestinal tract. Image-enhanced endoscopy has recently been introduced in the field of otorhinolaryngology, and the usefulness of this technique as compared to conventional white-light endoscopy has been reported.

Although esophageal carcinoma and gastric carcinoma share few risk factors, smoking is said to be a common risk factor for both esophageal carcinoma and gastric carcinoma. The high prevalence of atrophy of the gastric mucosa due to Helicobacter pylori infection and the high morbidity rate of gastric carcinoma due to environmental factors in Japan may exert a great influence to incidence of concomitant gastric lesion with esophageal cancer.

As a part of double/multiple carcinomas, carcinomas developing in the gastric tube after esophageal reconstruction pose another important problem.

When carrying out pretreatment examination of patients with esophageal carcinoma, due caution is necessary, because double carcinomas frequently involve areas that may greatly influence the choice of the particular therapeutic strategies used. 


\section{Follow-up observation after treatment of esophageal carcinoma}

The purposes of follow-up observation after treatment of esophageal carcinoma are (1) early detection and early treatment of recurrence and (2) early detection and early treatment of multiple esophageal carcinomas and double carcinomas involving other organs. In addition, follow-up observation is important from the point of view of general management of the patient after treatment and maintaining the patient's QOL.

The methods used for follow-up observation after treatment of esophageal carcinoma depend on the initial treatment employed and the stage of the disease at the time of the initial treatment. The patient follow-up is important for possible recurrence, bearing in mind the fact that early detection and early treatment of recurrence may allow prolongation of life. It is also important to exercise caution for the development of metachronous multiple esophageal carcinoma or metachronous multiple carcinoma of other organs, such as gastric carcinoma or head and neck carcinoma. Formulation of an effective follow-up protocol based on general agreements by many doctors in Japan and verification of its efficacy are required.

Follow-up observation after endoscopic resection

Because the indications and types of additional treatment after endoscopic resection vary, and a substantial number of patients undergo follow-up observation alone, there is no standard method for follow-up observation. Local recurrence after endoscopic resection is often seen within 1 year after the initial treatment, but may also occur after 2-3 years. Therefore, long-term follow-up is necessary. Esophageal endoscopy with iodine staining is mainly used for the detection of local recurrence. Although some reports propose examinations at 6-month intervals, other reports recommend examinations at 3-month intervals during the first year after resection. Patients who undergo fractional resection or who have multiple zones of negative iodine staining require more detailed endoscopic examination of the esophagus. Lymph node recurrence and organ recurrence may be found after 2-3 years; therefore, periodic long-term observation is necessary.

Patients should be followed up at 6- to 12-month intervals by cervical and abdominal ultrasonography, thoracoabdominal contrast CT, and/or EUS. Follow-up methods after endoscopic resection suggested by JCOG0508 study (phase II study on efficacy of combined treatment of endoscopic mucosal resection [EMR] and chemoradiotherapy for clinical Stage I esophageal carcinoma [T1NOM0]) included clinical examination, cervical to abdominal contrastenhanced CT, and measurement of the serum levels of the tumor marker SCC every 4 months until 3 years after EMR.
Follow-up observation after radical surgery

Recurrence after radical surgery has been reported to occur in 28-47 \% of patients in Japan. A recurrence rate of $50 \%$ or more is not rare in reports from Europe and North America. Among patients with recurrence, the timing of recurrence is within 1 year after surgery in $54-79 \%$ of patients and within 2 years after surgery in $80-98 \%$ of patients. Although rare, recurrence after more than 2 years can also occur, necessitating caution. The mode of recurrence may be lymph node metastasis, local recurrence, organ metastasis, or disseminated recurrence; a combination of these is also encountered frequently.

Currently, the actual follow-up protocol employed after radical surgery for esophageal carcinoma is left to the discretion of the treating facility. There are no reports of the benefit of regular follow-up observations or of effective methods of follow-up observation. Examination for recurrence basically consists of head and neck US, thoracoabdominal contrastenhanced CT, and bone scintigraphy. At many facilities, examination by US or CT is repeated every 3-6 months, often with some variations of the intervals according to the degree of progression and the number of years elapsed after surgery. Follow-up is generally carried out for 5 years, although some facilities continue to follow up their patients for 10 years. During the implementation of diagnostic imaging, many facilities add examination by interview, physical examination, and measurement of tumor markers.

Follow-up observation after definitive chemoradiotherapy

Although CT and esophageal endoscopy are usually employed for follow-up observation after definitive chemoradiotherapy, there have been no reports providing evidence for establishing the optimal frequency for such examinations or the duration of follow-up. In most cases, these examinations are performed at 3-4 weeks after the end of chemoradiotherapy and after the end of each course of additional chemotherapy. Thereafter, followup examinations are generally carried out every 3 months during the first year after therapy, and every 4-6 months from the second year onward after therapy. Residual carcinoma or recurrence after chemoradiotherapy is found frequently in the primary focus in the esophagus or in the regional lymph nodes, and in most cases recurrence occurs within 1-2 years after the start of therapy (see "Chemoradiotherapy").

After definitive chemoradiotherapy for esophageal carcinoma, observation for possible late adverse events related to radiotherapy such as radiation pneumonitis, pleural effusion, and pericardial effusion is necessary, in addition to examinations for recurrence. These aforementioned disorders may cause deterioration of a patient's QOL, and 
radiotherapy-related late toxicity may even lead to death (see "Radiotherapy").

Surveillance for metachronous multiple esophageal carcinomas and multiple carcinomas arising from other organs

Esophageal carcinoma is relatively frequently accompanied by metachronous multiple esophageal carcinoma. In addition, the occurrence of metachronous carcinomas in other organs, such as gastric carcinoma and cancer of the head and neck region, is not rare. Metachronous carcinoma of other organs has been reported as the predominant cause of postoperative death in $\mathrm{pNO}$ patients. Bearing this in mind, it is necessary to perform endoscopic examination of the upper gastrointestinal tract and to observe the areas from the pharynx to the entire esophagus (remaining esophagus in resected cases) and the stomach regularly and carefully. Surveillance for the development of colorectal carcinoma or other carcinomas is also necessary.

\section{Treatment of recurrent esophageal carcinoma}

\section{Summary}

The initial treatment for esophageal carcinoma is selected from a wide variety of options, including endoscopic treatment, radical surgery, and definitive chemoradiotherapy. Therefore, treatment of recurrent esophageal carcinoma should be determined according to the modality selected for the initial treatment. In addition, treatment of recurrent carcinoma varies according to the type of recurrence, i.e., lymph node metastasis, local recurrence, distant organ metastasis, or the combination of these. The general condition of the patient at the time of recurrence also exerts influence on the selection of treatment. Recurrence is not rare even in patients in whom the initial treatment has been properly implemented. Large-scale clinical trials to clarify issues related to treatment of recurrence are difficult to conduct. Recurrent carcinoma may be curable depending on the type of recurrence, and aggressive treatment may be desirable. Treatment, however, is often aimed at suppression of tumor aggravation and improvement of the QOL.

\section{Treatment of recurrence after endoscopic resection}

Although local recurrence after endoscopic mucosal resection most often occurs within 1 year after the initial treatment, it may even occur after 2-3 years in some cases. In recent years, the indications for endoscopic resection have been extended from the aspect of clinical research. The indications and types of additional treatment after endoscopic resection are variable, and quite a number of patients are followed up without any additional treatment.

\section{Treatment of recurrence after radical surgery}

Recurrence after radical surgery has been reported to occur in $28-47 \%$ of patients in Japan. Reports of recurrence rates of $50 \%$ or more are not rare from Europe and North America. In relation to the mode of recurrence, lymph node or local recurrence is found in $22-68 \%$ of patients, and distant organ metastasis in $12-51 \%$ of patients; the two types of recurrences have been reported to occur in combination in $7-27 \%$ of patients. Lymph node recurrence usually involves the cervical or superior mediastinal lymph nodes, and distant organ recurrence most frequently involves the lung, followed by the liver, bone and brain, in descending order of frequency. Metastasis to the small intestine or colon has also been reported.

The survival rate of patients with recurrence after radical resection of esophageal carcinoma is extremely poor, with the median survival time from the diagnosis of recurrence reported to be 5-10 months. However, long-surviving cases and cases of complete cure do exist; therefore, aggressive treatment is desirable.

Treatment of recurrence after radical resection of esophageal carcinoma is selected on the basis of the site, type, and extent of recurrence. Treatment also depends on the general condition of the patient at the time of recurrence, whether the recurrence is within or outside the scope of surgical manipulation, and whether or not the patient has received radiation pre- or postoperatively. Therefore, there is little data on the treatment results from a large number of patients with various clinical conditions.

\section{Treatment of recurrence in cases showing $C R$ after definitive chemoradiotherapy}

It has become more common in recent years to adopt definitive chemoradiotherapy as the initial treatment, not only for cases of unresectable esophageal carcinoma, but also for those with resectable esophageal carcinoma. Although this therapy yields a relatively high rate of CR, recurrences, including local ones, are frequently seen (see "Chemoradiotherapy").

\section{Palliative medicine}

Summary

Although palliative care should be provided in all fields of cancer, a decrease in the patient's QOL is particularly common in patients with esophageal carcinoma, caused by the 
difficulty in swallowing, malnutrition, and/or cough due to fistula formation. Consideration of procedures for symptom relief and maintenance and improvement of the QOL is required from the initial stages of treatment. However, selection of such procedures is currently left to the discretion of the treating institution. Close investigation of this issue would be desirable in the future. All health-care providers should acquire the basic knowledge and skills involved in the field of palliative medicine.

According to the WHO (2002), palliative care is defined as "an approach that improves the quality of life of patients and their families facing problems associated with lifethreatening illness, through the prevention and relief of suffering by early identification and impeccable assessment and treatment of pain and other problems, physical, psychosocial and spiritual." Palliative care should ideally begin when a patient is diagnosed as having cancer. This type of care is needed by all cancer patients and is provided in daily clinical practice. Palliative care requires a team approach that includes not only the doctors in charge and nurses, but also psycho-oncology specialists, pharmacists, social workers, and physical therapists. It has been pointed out that in particular, the role of a specialist nurse as a team leader is important in the palliative care of patients with esophageal carcinoma.

It is not rare, particularly in cases of esophageal carcinoma, that the patient has decreased QOL from the time of diagnosis because of difficulty in swallowing and malnutrition due to esophageal stenosis, cough due to mis-swallowing, or a fistula and chest pain due to the tumor. It is important to provide palliative care and treatment for the purpose of maintaining or improving QOL in parallel with the initial treatment that is aimed at cure of the disease.

Important issues in the palliative care of patients with end-stage esophageal carcinoma include difficulty in swallowing due to esophageal stenosis and the resultant malnutrition, the symptoms arising from airway stenosis and fistula formation to the airway, and cachexia and other symptoms due to distant metastasis and hypercalcemia. Among these, relief of symptoms arising from esophageal stenosis, airway stenosis, or fistula formation may be attempted by palliative treatments such as radiotherapy, chemoradiotherapy, esophageal stent insertion, airway stent insertion, and esophageal bypass (see "Radiotherapy" and "Chemoradiotherapy").

Gastrostomy or enterostomy as well as intravenous hyperalimentation may be performed to deal with malnutrition. These palliative treatments are typically employed for patients with esophageal carcinoma. Correct decisions as to the method and timing of implementation of these treatments are critical in the provision of palliative care for patients with end-stage esophageal carcinoma.
However, there have been few large-scale studies that have evaluated the efficacy and safety of various treatments and procedures in palliative medicine for patients with esophageal carcinoma. There have been no studies on the possibility of radiotherapy or chemotherapy providing survival advantage over best supportive care. However, it is a fact that in the actual clinical setting, a certain proportion of patients who have undergone these treatments have shown marked improvement in their QOL. Health-care providers should be skilled in palliative treatments and procedures specific to esophageal carcinoma, and the appropriate treatments should be employed proactively after obtaining informed consent from the patients.

In addition, health-care providers who are engaged in the treatment of esophageal carcinoma often encounter fatal conditions, such as acute respiratory arrest due to airway obstruction or massive hematemesis due to perforation into the aorta. In many cases, rescue of the patient is difficult once these events occur. It is important to give adequate explanation in advance about the possible occurrence of such events, particularly to the patients' families. Because the patients and their families have to live with the fear of sudden death or sudden change in clinical condition, provision of psychological support and mental care to both are indispensable. To treat carcinomarelated pain, procedures described in the Clinical Guideline for Pharmacological Management of Cancer Pain issued by the Japanese Society for Palliative Medicine are recommended.

\section{Therapeutic efficacy and guidelines in Europe and North America: including the results of prognostic studies based on national registries}

\section{Summary}

Unlike the situation in Japan, in Europe and North America, adenocarcinomas originating in the lower esophagus account for a large proportion of esophageal lesions. Therefore, the methods of treatment and their results are not necessarily comparable to those in Japan.

A simple comparison of endoscopic treatments in Japan and Western countries is precluded by differences in the criteria for selection of suitable candidates. There are no well-established guidelines.

Transhiatal esophagectomy is common, reflecting the increase in the frequency of lower esophageal adenocarcinoma. The extent of lymph node dissection is often restricted to the middle and lower mediastinum. Although there are no large differences from Japan in terms of surgical indications in relation to the disease stage, the surgical 
results have not been satisfactory in Europe and North America.

The reported efficacy of neoadjuvant chemotherapy varies between Europe and North America. US Guidelines restrict neoadjuvant chemotherapy to carcinomas of the lower esophagus and the esophagogastric junction, and recommend neoadjuvant chemoradiotherapy for carcinoma arising in other parts of the esophagus. The England/Wales and Scotland, guidelines recommend 2 courses of neoadjuvant chemotherapy for cases with resectable disease, but do not recommend neoadjuvant chemoradiotherapy.

In regard to non-surgical treatment, as chemoradiotherapy has been shown to yield better results than radiation monotherapy; guidelines published in Europe and North America both recommend chemoradiotherapy as do those published in Japan.

There are differences in the epidemiology of esophageal carcinoma between Japan and Europe/North America, which make it impractical to simply compare the methods and results of treatment between these regions. In regard to the histologic type, squamous cell carcinoma accounts for more than $90 \%$ of all cases of esophageal carcinoma in Japan, whereas adenocarcinoma accounts for more than $50 \%$, and squamous cell carcinoma for less than $40 \%$ of cases in Europe and North America. As for the location of the tumor, tumors arising in the middle thoracic esophagus are reported as the most frequent, accounting for more than $50 \%$ of all cases in Japan, whereas lesions arising in the lower thoracic esophagus are reported to account for more than $50 \%$ of the cases in Europe and North America. The past two decades have seen an increase in the frequency of adenocarcinoma in Western countries, and it is reported that Barrett's esophagus developing from obesity and GERD have been reported as background factors for this increase. Therefore, these factors account for the differences in therapeutic strategies and treatment results between Japan and the US/Europe.

Guidelines for the diagnosis and treatment of esophageal carcinoma have also been published in some Western countries. These include 4 comprehensive guidelines, i.e., 2 guidelines from the USA, 1 from the UK, and another from Scotland. In the USA, Physician Data Query (PDQ) from the National Cancer Institute (NCI) and information from the National Comprehensive Cancer Network (NCCN) are available on the Internet, and the information is updated continually, with data added from the latest literature. In particular, the NCCN guidelines provide an algorithm to facilitate selection of the appropriate treatment. The strength of recommendation is categorized according to the level of evidence. In the guidelines published in England/ Wales and Scotland, the levels of evidence are specified as in the Japanese guidelines. They provide specific information on the epidemiology and pathogenesis, perioperative management, and postoperative complications as well as on the palliative treatment of esophageal carcinoma.

\section{Endoscopic treatment}

Although there are reviews on endoscopic treatment from Europe and North America, they differ from reports published in Japan in those cases of high-grade dysplasia are included as target lesions, and photodynamic therapy (PDT) is also included as a type of treatment. Therefore, a simple comparison with reports from Japan is not possible. The reported 5-year survival rate after EMR is $87.7 \%$ in Japan, according to the national statistics published in 2002.

It is rare in Western countries for esophageal carcinoma to be detected at an early stage; therefore, EMR is not commonly performed. Therefore, guidelines regarding endoscopic treatment are limited. Although the NCCN guidelines recommend EMR for mucosal carcinoma (Tis or T1a), the NCI guidelines recommend surgery for Stage 0 lesions. In the Scottish guidelines, EMR is recommended for carcinomas confined to the mucosal layer. The England/Wales guidelines contain no description of endoscopic resection.

\section{Surgery}

In the USA, transhiatal esophagectomy without thoracotomy is regarded as the standard surgical treatment technique for esophageal carcinoma for the following reasons: the results of resection are poor due to the prevalence of advanced carcinoma; surgical complications are frequent because of the high rate of high risk patients; carcinoma in the lower esophagus is frequent; no difference in the recurrence-free survival has been reported between transhiatal esophagectomy and subtotal esophagectomy via right thoracotomy accompanied by lymph node dissection and complications are less frequent with the former procedure. The extent of lymph node dissection is often restricted to the middle and lower mediastinum, and dissection covering all the three regions (cervical, thoracic, and abdominal) is not generally performed. Table 7 shows the results of randomized controlled trials of surgical treatment of esophageal carcinoma reported in and after 1990, and the 2002 Japanese national registry data. Although there are variations in the stages considered for resection among overseas studies, the reported 5-year survival rate is generally about $25 \%$ or less, being significantly different from the corresponding rate of $44.1 \%$ in all cases treated by resection in Japan.

According to the NCCN guidelines, surgery is indicated for Stage 0-III or resectable Stage IVA carcinoma of the esophagus. As for cases of cervical esophageal carcinoma, the NCCN guidelines state that definitive 


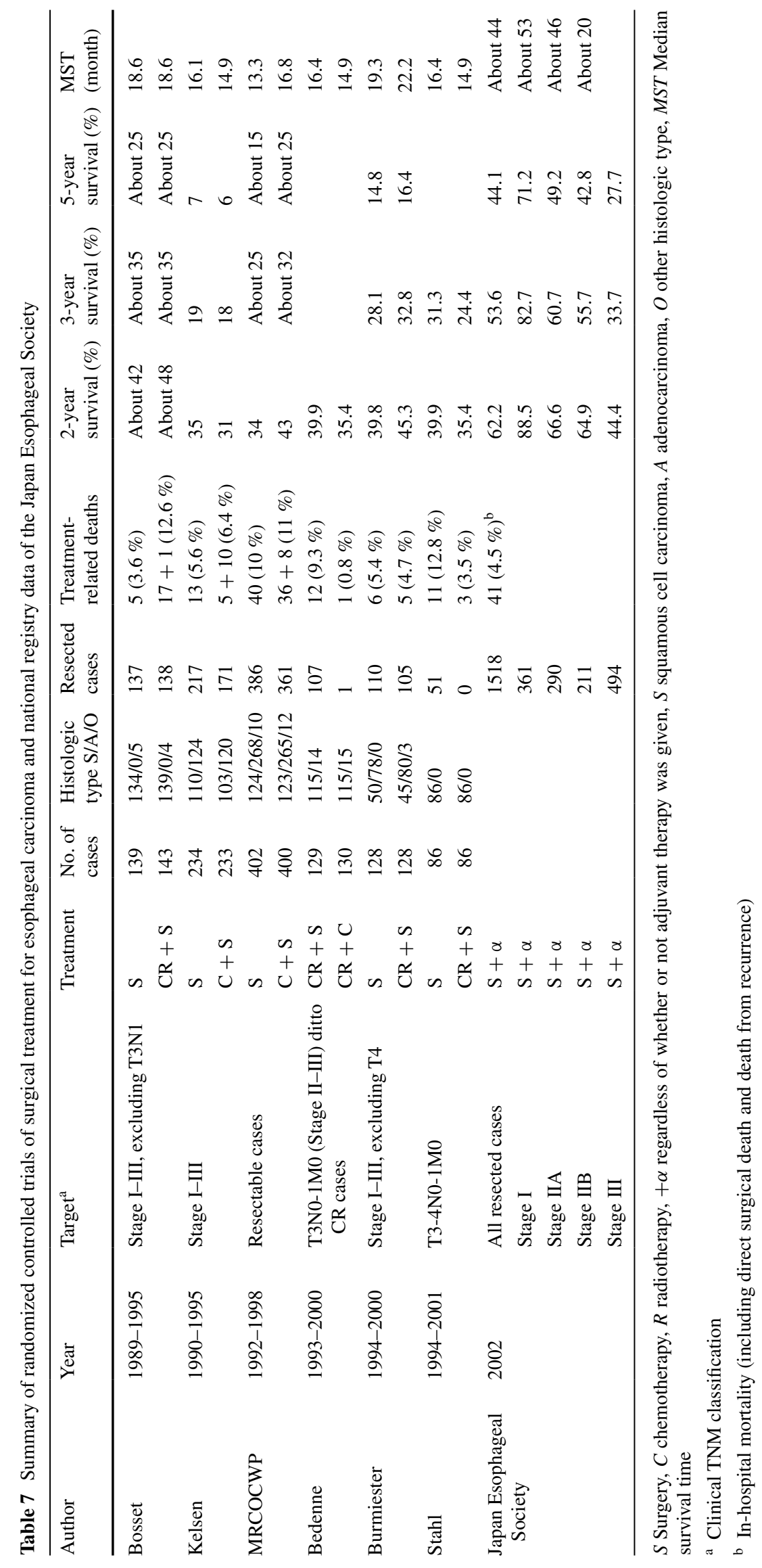


chemoradiotherapy should be administered for carcinomas in this region and those located less than $5 \mathrm{~cm}$ from the cricopharynx, with no consideration of surgery.

\section{Preoperative and postoperative adjuvant therapy}

Preoperative adjuvant therapy A meta-analysis of several randomized controlled trials performed in Europe and North America to examine the usefulness of neoadjuvant chemotherapy revealed no consistent benefit of neoadjuvant chemotherapy on survival. Thus, the efficacy of neoadjuvant chemotherapy for patients with resectable disease (T1-3N0, 1M0, UICC classification, 2002 edition) remains unclear. In addition, a metaanalysis reported in Australia in 2007 concluded that neoadjuvant chemoradiotherapy may have additional benefit in cases of esophageal adenocarcinoma, but is not effective for patients with squamous cell carcinoma, emphasizing the need to choose adjuvant therapy according to the histologic type of the disease.

According to the NCCN guidelines, preoperative therapy is indicated for cases with $\mathrm{T} 1 \mathrm{~b}, \mathrm{~N} 1, \mathrm{~T} 2$ to resectable T4, and resectable Stage IVA disease. Neoadjuvant chemotherapy is restricted to cases of adenocarcinoma located in the lower esophagus or the esophagogastric junction. Lesions in other parts of the esophagus are described as indications for neoadjuvant chemoradiotherapy, with the recommended drugs specified by category. The England/ Wales and Scotland guidelines do not recommend neoadjuvant chemoradiotherapy, although they state that adjuvant chemotherapy with 2 courses of cisplatin +5 -FU should be considered for cases with resectable disease.

Postoperative adjuvant therapy The NCCN guidelines recommend postoperative chemotherapy only for patients who have received neoadjuvant chemotherapy. Chemoradiotherapy is recommended for T2-3N0-1 adenocarcinoma or N1 adenocarcinoma cases with R0 resection. Some R1-2 cases may also be included. The Scottish guidelines do not recommend either postoperative chemotherapy or chemoradiotherapy, based on the results of randomized controlled trials for the former and the lack of data for the latter. The England/Wales guidelines also do not recommend postoperative chemotherapy.

\section{Chemoradiotherapy}

With regard to non-surgical treatment, conventional guidelines recommend the use of chemoradiotherapy, based on reports that chemoradiotherapy yielded better results when concurrent chemoradiotherapy was compared with radiation monotherapy. According to the 2002 national registry of the Japan Esophageal Society, the 5-year survival rate was $15.1 \%$ in patients who received radiation monotherapy, whereas it was $22.9 \%$ in those treated by chemoradiotherapy. The corresponding rates by disease stage were 32.5 vs. $52.0 \%$ for Stage I-IIA cases and 4.2 vs. $14.9 \%$ for Stage IIB-IVB cases, indicating the superiority of chemoradiotherapy over radiation monotherapy. The protocol recommended by the Radiation Therapy Oncology Group that is commonly employed in Europe and North America consists of irradiation using the multiple field technique at a total dose of 50.4 Gy administered in 28 fractions, with the exposure field covering the region within $5 \mathrm{~cm}$ above and below the tumor. This regimen is based on the results of a randomized controlled trial that found no difference in the survival period between standard-dose (50.4 Gy) and high-dose (64.8 Gy) chemoradiotherapy, and reached a negative conclusion about the benefit of increasing the total radiation dose. The NCCN guidelines specify that the radiation dose should be 50-50.4 Gy.

Acknowledgments We express our heartfelt condolences for the death of Professor Hiroya Takiuchi. We extend his gratitude for his contribution to these guidelines.

Ethical Statement All procedures followed were in accordance with the ethical standards of the responsible committee on human experimentation (institutional and national) and with the Helsinki Declaration of 1964 and later versions. This article does not contain any studies with human or animal subjects performed by any author(s).

Conflict of interest Funds to support the development of these guidelines were provided by the Japan Esophageal Society and the Ministry of Health, Labour and Welfare Scientific Research (Study on the appropriate development and publication of guidelines for the diagnosis and treatment while maintaining full disclosure: Hirata's study group). Prof. Kitagawa has received grants from Taiho Pharmaceutical CO., LTD., Chugai Pharmaceutical CO., LTD., Kyowa Hakko Kirin CO., LTD., Merck Serono CO., LTD., Ono Pharmaceutical CO., LTD., Novartis Pharma CO., LTD., Yakult Honsha CO., LTD., Bristol-Myers Squibb Daiichi Sankyo CO., LTD., Shionogi CO., LTD., Terumo Corporation, Torii Pharmaceutical Co., Ltd., AstraZeneca CO., LTD. Other authors have no conflict of interest to declare with profit making or nonprofit organizations or providers of medical products and medical supplies. The Ethics Committee and Administrative Board of the Japan Esophageal Society required members of the Committee to Develop Guidelines for the Treatment of Carcinoma of the Esophagus and the Guideline Evaluation Committee to self-report any potential conflict of interest. 